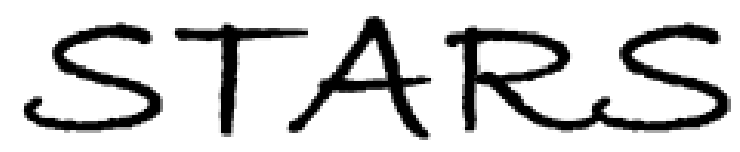

University of Central Florida

STARS

$1-1-2012$

\title{
Cassini UVIS observations of Titan nightglow spectra
}

Joseph M. Ajello

Robert A. West

Jacques Gustin

Kristopher Larsen

A. Ian F. Stewart

See next page for additional authors

Find similar works at: https://stars.library.ucf.edu/facultybib2010

University of Central Florida Libraries http://library.ucf.edu

This Article is brought to you for free and open access by the Faculty Bibliography at STARS. It has been accepted for inclusion in Faculty Bibliography 2010 s by an authorized administrator of STARS. For more information, please contactSTARS@ucf.edu.

\section{Recommended Citation}

Ajello, Joseph M.; West, Robert A.; Gustin, Jacques; Larsen, Kristopher; Stewart, A. Ian F.; Esposito, Larry W.; McClintock, William E.; Holsclaw, Gregory M.; and Bradley, E. Todd, "Cassini UVIS observations of Titan nightglow spectra" (2012). Faculty Bibliography 2010s. 2205.

https://stars.library.ucf.edu/facultybib2010/2205

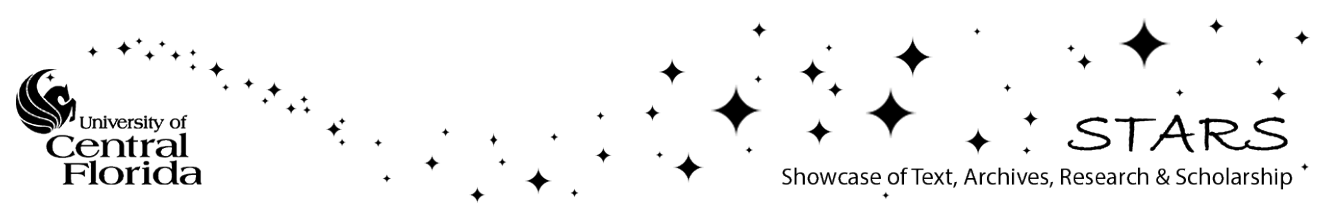




\section{Authors}

Joseph M. Ajello, Robert A. West, Jacques Gustin, Kristopher Larsen, A. Ian F. Stewart, Larry W. Esposito, William E. McClintock, Gregory M. Holsclaw, and E. Todd Bradley 


\title{
Cassini UVIS observations of Titan nightglow spectra
}

\author{
Joseph M. Ajello, ${ }^{1}$ Robert A. West, ${ }^{1}$ Jacques Gustin, ${ }^{2}$ Kristopher Larsen, ${ }^{3}$ \\ A. Ian F. Stewart, ${ }^{3}$ Larry W. Esposito, ${ }^{3}$ William E. McClintock, ${ }^{3}$ Gregory M. Holsclaw, ${ }^{3}$ \\ and E. Todd Bradley ${ }^{4}$
}

Received 7 May 2012; revised 24 September 2012; accepted 19 October 2012; published 22 December 2012.

[1] In this paper we present the first nightside EUV and FUV airglow limb spectra of Titan showing molecular emissions. The Cassini Ultraviolet Imaging Spectrograph (UVIS) observed photon emissions of Titan's day and night limb-airglow and disk-airglow on multiple occasions, including during an eclipse observation. The 71 airglow observations

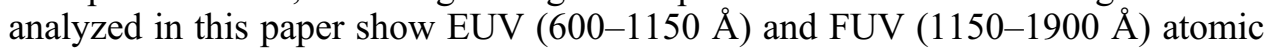
multiplet lines and band emissions arising from either photoelectron induced fluorescence and solar photo-fragmentation of molecular nitrogen $\left(\mathrm{N}_{2}\right)$ or excitation by magnetosphere plasma. The altitude of the peak UV emissions on the limb during daylight occurred inside the thermosphere at the altitude of the topside ionosphere (near $1000 \mathrm{~km}$ altitude). However, at night on the limb, a subset of emission features, much weaker in intensity, arise in the atmosphere with two different geometries. First, there is a twilight photoelectron-excited glow that persists with solar depression angle up to 25-30 degrees past the terminator, until the solar XUV shadow height passes the altitude of the topside ionosphere (1000-1200 km). The UV twilight glow spectrum is similar to the dayglow but weaker in intensity. Second, beyond $120^{\circ}$ solar zenith angle, when the upper atmosphere of Titan is in total XUV darkness, there is indication of weak and sporadic nightside UV airglow emissions excited by magnetosphere plasma collisions with ambient thermosphere gas, with similar $\mathrm{N}_{2}$ excited features as above in the daylight or twilight glow over an extended altitude range.

Citation: Ajello, J. M., R. A. West, J. Gustin, K. Larsen, A. I. F. Stewart, L. W. Esposito, W. E. McClintock, G. M. Holsclaw, and E. T. Bradley (2012), Cassini UVIS observations of Titan nightglow spectra, J. Geophys. Res., 117, A12315, doi:10.1029/2012JA017888.

\section{Introduction}

[2] There are four nitrogen-bearing atmospheres in the solar system: Titan, Triton, Earth and Pluto. The complex UV airglow signatures of $\mathrm{N}_{2}$ and its dissociation products from the mesosphere and thermosphere are the principal means of studying the energy input to a planetary atmosphere. The response of the Titan upper atmosphere to both the solar cycle variation of the solar extreme ultraviolet (XUV) and local magnetosphere particle injection is a prime focus of the Cassini Mission. Based on Cassini in situ data, Strobel

\footnotetext{
${ }^{1}$ Jet Propulsion Laboratory, California Institute of Technology, Pasadena, California, USA.

${ }^{2}$ Laboratoire de Physique Atmosphérique et Planétaire, Université de Liège, Liège, Belgium.

${ }^{3}$ Laboratory for Atmospheric and Space Physics, University of Colorado Boulder, Boulder, Colorado, USA.

${ }^{4}$ Department of Physics, University of Central Florida, Orlando, Florida, USA

Corresponding author: J. M. Ajello, Jet Propulsion Laboratory, California Institute of Technology, Pasadena, CA 91109, USA. (joseph.m.ajello@jpl.nasa.gov)

(C2012. American Geophysical Union. All Rights Reserved. 0148-0227/12/2012JA017888
}

and $C u i$ [2012] have shown that the globally averaged magnetosphere energy input to Titan's upper atmosphere is almost always smaller than the solar XUV.

[3] Photoelectron excitation dominates the Titan dayside airglow spectrum [Stevens et al., 2011]. Energetic protons $\left(\mathrm{H}^{+}\right)$ and $\mathrm{O}^{+}$ions have been observed by Cassini/Magnetosphere Imaging Instrument (MIMI) and thermal and suprathermal ions are studied by Cassini Plasma Spectrometer (CAPS) in Saturn's outer magnetosphere. These particles can precipitate into Titan's atmosphere where they deposit energy, generating airglow emissions and driving ionospheric chemistry for both the lower atmosphere ionosphere and topside ionosphere [Sittler et al., 2010a, 2010b; Cravens et al., 2008; Ledvina et al., 2005; Lavvas et al., 2011; Westlake et al., 2011; Fulchignoni et al., 2005; Gan et al., 1992; Smith et al., 2009; Shah et al., 2009]. The energies of the upstream magnetosphere plasma ions interacting with Titan's thermosphere $(400-1400 \mathrm{~km})$ extend over a large energy range from $0.1 \mathrm{keV}$ to $4 \mathrm{MeV}$ for protons, from $2 \mathrm{keV}$ to $4 \mathrm{MeV}$ for oxygen ions [Cravens et al. 2008] and $10 \mathrm{eV}$ to $10 \mathrm{keV}$ for electrons [Cravens et al., 2008, 2009]. Either energetic electron or proton interactions with ambient neutral species can be a significant source of nightglow emissions [Ajello et al., 2010, 2011a, 2011b]. 
[4] Titan is lacking significant intrinsic magnetization, and is normally immersed in Saturn's rapidly rotating magnetosphere allowing direct access of the co-rotating plasma to the thermosphere region [Westlake et al., 2011; De La Haye et al., 2007]. Saturn's magnetospheric interaction with Titan from recent results of CAPS and Cassini magnetometer (MAG) show the upstream flow appears composed of intense light ions (protons $\left(\mathrm{H}^{+}\right)$and $\mathrm{H}_{2}^{+}$) and the heavier ion $\mathrm{O}^{+}$ [Sittler et al., 2010b]. The altitude deposition ranges of protons and $\mathrm{O}^{+}$(the second most abundant ion in the co-rotating plasma) indicates an extended nighttime ionosphere layer excitation source below $1000 \mathrm{~km}$ [Cravens et al., 2008] and verified recently by Kliore et al. [2008] by the radio occultation experiment discovery of the lower sporadic ionosphere. According to Kliore et al. [2008], there is a ionospheric peak for one of the polar observations in the region of $500-600 \mathrm{~km}$. The energy deposition rates of the upper atmosphere from 600 to $1500 \mathrm{~km}$ by precipitating ions, $\mathrm{H}^{+}$and $\mathrm{O}^{+}$have been modeled by Westlake et al. [2011] using Cassini Ion and Neutral Mass Spectrometer (INMS) data. The observations show that Titan's $\mathrm{N}_{2}$ global thermosphere distribution quickly responds to both $\mathrm{O}^{+}$energy deposition that peaks at $1100 \mathrm{~km}$ and $\mathrm{H}^{+}$energy deposition that peaks at $700 \mathrm{~km}$.

[5] To date we have published UV results from two Cassini Titan passes. Analyses of the 13 December 2004 (13Dec04, TB) and 22 June 2009 (22 June 09, T57) Titan dayside observations of the extreme ultraviolet (EUV: 900 $1140 \AA$ ) and the far ultraviolet (FUV: $1150-1900 \AA$ ) disk and limb spectra from the Ultraviolet Imaging Spectrometer (UVIS) on Cassini have been recently published. These two observation dates were chosen for analysis because of particularly high $\mathrm{S} / \mathrm{N}$ in their dayglow spectra. We refer to the UVIS publications of these two observations as Paper 1 [Ajello et al., 2007], Paper 2 [Ajello et al., 2008], and Paper 3 [Stevens et al., 2011], respectively. Model spectral fits to the Titan FUV disk dayglow disk spectrum of 13Dec04 described in Paper 2 and to the Titan FUV limb spectrum of 22June09 in Paper 3 revealed that Titan's FUV dayglow emission is attributed to five main sources: (1) the LymanBirge-Hopfield (LBH) band system of $\mathrm{N}_{2}\left(\mathrm{a}^{1} \Pi_{\mathrm{g}}-\mathrm{X}^{1} \sum_{g}^{+}\right)$ due to photoelectrons produced by solar XUV on $\mathrm{N}_{2}$ [Young et al., 2010; Ajello and Shemansky, 1985; Ajello et al., 2010]; (2) N I multiplets primarily due to solar photodissociative ionization (PDI) excitation of $\mathrm{N}_{2}$ [Meier et al., 1991; Samson et al., 1991; Mumma and Zipf, 1971]; (3) the H Ly $\alpha$ emission line excited mainly by solar fluorescence at $1215.6 \AA ;(4)$ the $\mathrm{N}_{2}$ Vegard-Kaplan (VK) band system $\left(\mathrm{A}^{3} \sum_{\mathrm{u}}^{+} \rightarrow \mathrm{X}^{1} \sum_{g}^{+}\right)$ peaking near $900 \mathrm{~km}$ altitude also from photoelectrons [Stevens et al., 2011; Shemansky, 1969]; and (5) reflected sunlight, predominantly arising from altitudes below $800 \mathrm{~km}$ and wavelengths longward of $1500 \AA$. The first four sources are the same as observed in the thermosphere of the Earth by the Far Ultraviolet Spectrographic Explorer (FUSE) instrument and other earth-orbiting spacecraft [Bishop and Feldman, 2003; Bishop et al., 2007; Broadfoot et al., 1997; Feldman et al., 2001; Morrison et al., 1990].

[6] The electronic excitations proceeding from the $X^{1} \sum_{g}^{+}$ ground-state to several closely spaced $(\sim 12-15 \mathrm{eV})$ states are the source of molecular emissions in the EUV observed by spacecraft, and have been discussed in Paper 1 for 13Dec04 and Paper 3 for 22June09. Two of the excited states, $\mathrm{b}^{1} \Pi_{u}$, b' $1 \Sigma$ are nominally valence in character and three others; $\mathrm{c}_{3}{ }^{1} \Pi_{\mathrm{u}}, \mathrm{o}_{3}{ }^{1} \Pi_{\mathrm{u}}$, and $c_{4}^{\prime}{ }^{1} \sum_{u}^{+}$are Rydberg states with principal quantum number $n=3$. Because of the presence of strong homogeneous and heterogeneous interactions they are referred to here as Rydberg-valence (RV) states. The spectrum is weakly to-strongly predissociated by an indirect mechanism involving multiple states of ${ }^{3} \Pi_{u}$ and ${ }^{1} \Sigma^{+}$symmetry, some of which are unbound. Because of this, the predissociation to emission branching ratio varies haphazardly from almost zero to unity, leading to significant emission and predissociation cross sections that are the main source of the observed EUV $\mathrm{N}_{2}$ and atomic $\mathrm{N}$ emissions by the UVIS [Lewis et al., 2005a, 2005b, 2008a, 2008b; Ubachs et al., 2001; Liu et al., 2005; Shemansky et al., 1995; Ajello et al., 1989; J. M. Ajello, A. N. Heays, B. R. Lewis and S. Gibson, Highresolution EUV spectrum of $\mathrm{N}_{2}$ by electron impact, submitted to The Astrophysical Journal Supplement, 2012].

[7] No levels of the $c_{3}{ }^{1} \Pi_{\mathrm{u}}$ and $\mathrm{o}_{3}{ }^{1} \Pi_{\mathrm{u}}$ states are expected to appear here in emission because they are very efficiently predissociated. The presence of emission from the $\mathrm{b}^{\prime}{ }^{1} \sum_{\mathrm{u}}^{+}$state, is inferred from the orbit TB analysis of Ajello et al. [2007] as there are no sharp and strong features from this transition [Ajello et al., 1989; submitted manuscript]. Further members of the $c_{3}{ }^{1} \Pi_{\mathrm{u}}$ and $c_{4}^{\prime}{ }^{1} \sum_{u}^{+}$Rydberg series with $\mathrm{n}>3$ were found to emit in the EUV, with high-resolution observations made by Roncin et al. [1998] and emission cross sections reported in Ajello et al. [2010; submitted manuscript].

[8] In this paper we analyze $71 \mathrm{limb}$ observations from 19 April, 2009 to 28 January, 2010 of Titan to search for darkside UV emissions and trace the variability in dayglow, twilight glow and nightglow emission intensities from the parent molecule $\mathrm{N}_{2}$, the major species of the upper atmosphere of Titan [De La Haye et al., 2007]. Some of the important geometric variables for strong locally occurring (latitude and altitude) UV dayglow are solar zenith angle (SZA) and associated target center phase angle (TPA), Solar $\mathrm{XUV}$, and minimum ray height (MRH) at the limb. Nightglow emissions, which can be generated throughout the thermosphere arising from interactions with the co-rotating magnetosphere from 400 to $1400 \mathrm{~km}$, indicate that the solarincidence-RAM angle (SRA or RAM) and Titan latitude are important for determining the side and latitude of Titan illuminated by the plasma stream. Using published literature Sittler et al. [2010a] estimated that particle energy input can rival the solar XUV; this estimate was recently proven erroneous by Strobel and Cui [2012]. These 71 observations in 2009-2010 took place at time of solar minimum as compared to Paper 1 observation of orbit $\mathrm{Tb}$ that occurred at time in 2004 that the UV solar flux was still weak in solar cycle 24, though not at solar minimum. We also analyze one Titan observation from 25 March 2008 near solar minimum that presented strong daylight and twilight glow emissions from both the limb and the disk. Rymer et al. [2009] point out that that low solar activity allows fewer magnetosheath plasma interactions with Titan. Low solar activity allows a larger magnetosphere as the solar wind pressure weakens compared to solar maximum. Most encounters in our time frame occur as the plasma sheet category [see Rymer et al., 2009].

[9] Many of these 71 observations contain daylight disk and nightside disk observations as well as the limb. The geometry for a limb observation is shown in Figure 1 to 


\section{UVIS OBSERVATION OF TITAN DARK DISK AND LIMB}

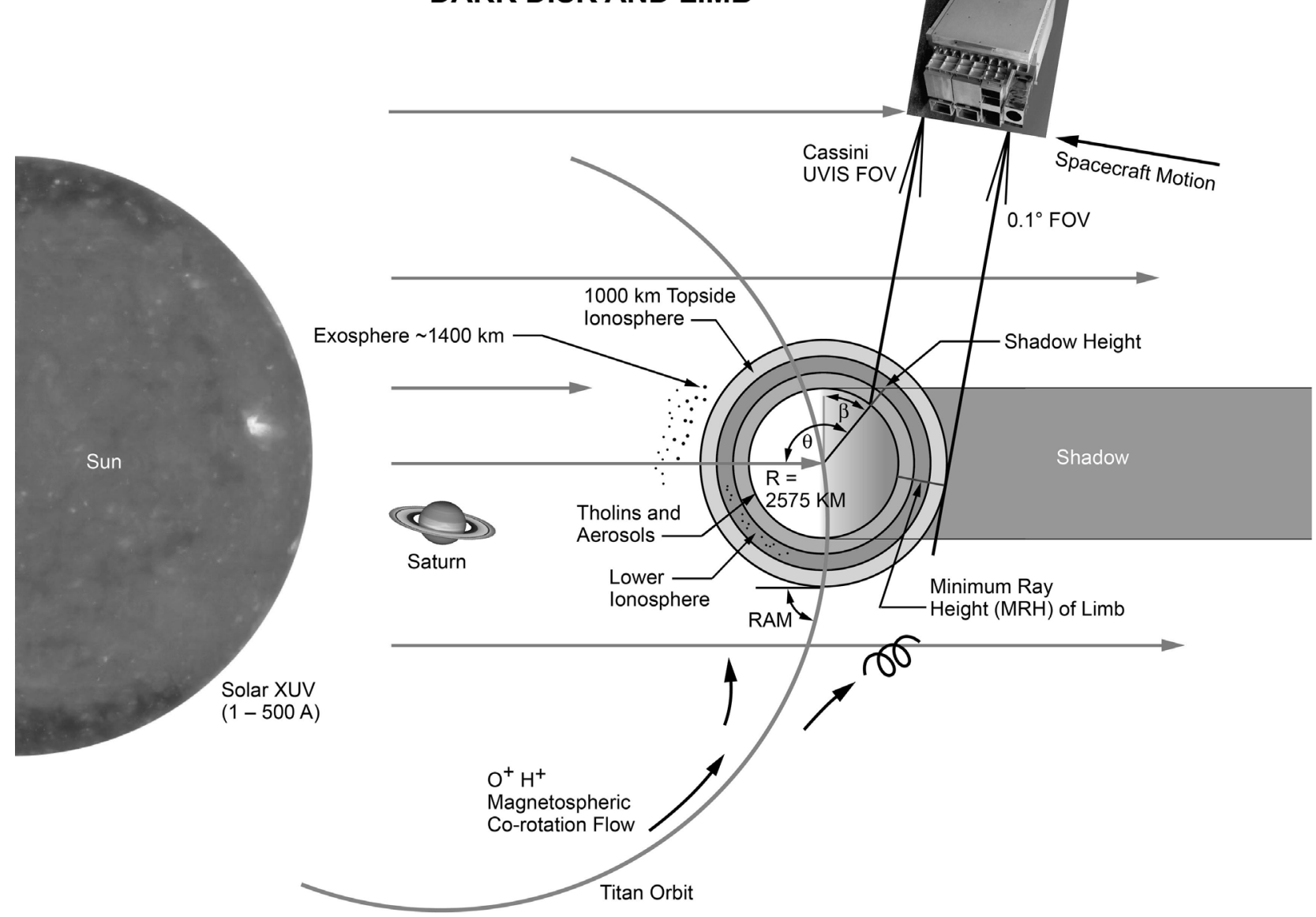

Figure 1. Nighttime viewing geometry of the UVIS limb and disk observations at a solar depression angle of $\beta$ through an atmosphere composed of a major gas, $\mathrm{N}_{2}(96 \%)$, and four distinct optical/UV layers: 1) haze (complex organic solids), surface to $400 \mathrm{~km} ; 2)$ lower thermosphere $(400-800 \mathrm{~km})$, including the lower ionosphere and a UV optically thick (10-1400 $\AA$ ) distribution of $\mathrm{CH}_{4}$ [Kliore et al., 2008]; 3) upper thermosphere (800-1400 km), including the topside ionosphere [Stevens et al., 2011]; and 4) the exosphere (above $1400 \mathrm{~km}$ ). The UVIS FUV (EUV) FOV for the low-resolution slit is $1.5 \mathrm{mrad} \times 60 \mathrm{mrad}$ $(2.0 \mathrm{mrad} \times 60 \mathrm{mrad})$ and is shown as $0.1^{\circ} \mathrm{FOV}$ in the diagram. The figure shows the four main layers of Titan's atmosphere illuminated by the Sun and interacting with the upstream magnetosphere flow of abundant ions: $\mathrm{H}^{+}$and $\mathrm{O}^{+}$at a RAM angle of $90^{\circ}$. The drawing indicates the presence of a shadow height an important altitude for twilight and nighttime observations. The shadow height is a function of solar depression angle. The MRH of a limb observation is also indicated.

remind us of the four atmosphere layers: 1) a distribution of haze (complex solid organics) or aerosols in the lower atmosphere (mesosphere) that persist up to an altitude of 400-500 km (or higher for detached haze layers [Tomasko and West, 2010], as we have shown in a set of altitudedependent FUV spectra demonstrating strong UV particulate, Rayleigh and Mie scattering, and reflection above 1500 $\AA$ in Sittler et al. [2010a, Figure 16.24]; 2) the lower thermosphere, where the temperature is about $\sim 170 \mathrm{~K}$ at $400 \mathrm{~km}$ and decreases with altitude $(400-800 \mathrm{~km})$ and the sporadic middle ionosphere appears [Kliore et al., 2008]; 3) the topside ionosphere from 800 to $1400 \mathrm{~km}$, which is the source region of the UV dayglow; and 4) the exosphere above $\sim 1400 \mathrm{~km}$. The haze or aerosol layer (and $\mathrm{CH}_{4}$ slant optical depth of unity in the XUV) may act as a secondary (tertiary) planetary absorbing surface for solar FUV (and XUV) radiation and for many years made Titan appear to be the largest moon in the solar system until Voyager observations.

[10] The shadow heights for the solar XUV and the solar FUV are important quantities for an extended atmosphere like Titan. For example, the ionosphere (airglow layer peak intensity at $1000 \mathrm{~km}$ altitude) represents $40 \%$ of the radius of the satellite $(2576 \mathrm{~km})$ compared to Earth with a $3 \%$ value for the ratio of the $\mathrm{E}, \mathrm{F}$ ionosphere region/airglow layer to the radius $(6378 \mathrm{~km})$. From Figure 1 we find that the shadow height $z_{\text {ion }}$ for three optical wavelength ranges measured from the satellite surface as a function of solar depression angle, $\left(\beta_{\text {opt }}\right)$ is given by

$$
\cos \left(\beta_{\text {opt }}\right)=\left(\mathrm{R}+\mathrm{h}_{\mathrm{opt}}\right) /\left(\mathrm{R}+\mathrm{z}_{\text {ion }}\right),
$$


where the subscript "opt" refers to a Titan radius $\left(\mathrm{R}+\mathrm{h}_{\mathrm{opt}}\right)$ altitude at the terminator where slant optical depth unity at the terminator occurs for near IR-visible wavelengths at minimum ray height altitude $\mathrm{h}_{\mathrm{opt}}=0 \mathrm{~km}, \mathrm{FUV}$ (wavelengths above $1400 \AA$ ) at altitude $\mathrm{h}_{\text {opt }}=300 \mathrm{~km}$, and FUV, EUV XUV (wavelengths below $1400 \AA$ ) at altitude $\mathrm{h}_{\text {opt }}=800 \mathrm{~km}$, respectively. The three optical shadow heights occur for hard surface conditions, particulate haze or high energy photons (wavelengths below $1400 \AA$ ) by an absorbing methane distribution (for solar rays, see also Ajello et al. [1973, Figure 1]). The upper altitude of the topside ionosphere is at about $z_{\text {ion }}=1200 \mathrm{~km}$ and allows us to determine for the three optical ranges where the ionosphere/airglow layer is in solar darkness (Paper 1). We find the three solar depression angles are approximately $47^{\circ}, 40^{\circ}$ and $26^{\circ}$, respectively. A UVIS limb airglow observation for a SZA line of sight above $116^{\circ}$ ensures darkness in the thermosphere and precludes till dawn diurnal enhancement of an ionosphere from solar XUV creation of photoelectrons. On the other hand, magnetosphere protons with energies above $30 \mathrm{keV}$ can deeply penetrate the atmosphere and excite the FUV from $\mathrm{N}_{2}$ (wavelengths above $1400 \AA$ ) for UV transmission at altitudes throughout the ionosphere, down to $500 \mathrm{~km}$ [Cravens et al., 2008; see also Sittler et al., 2010a, Figure 16.3].

\section{The Airglow Observations}

[11] The UVIS instrument includes a two-channel imaging spectrograph in the far ultraviolet (FUV: 1115-1912 $\AA$ ) and extreme-ultraviolet (EUV: 563-1182 Å) [Esposito et al., 2004]. The detector format for each channel is 1024 spectral pixels $\times 64$ spatial pixels, with 4 of the spatial pixels needed for engineering read-out information of the UVIS FUV (or EUV). The UVIS measurements will record spectral data for 60 pixels numbered 3-62 and referred to as $0-59$ in this paper. The Titan observations discussed here were taken with a spectral resolution of $5.0 \AA$ (low-resolution mode) and a rectangular field of view of $1.5(2.0) \times 60 \mathrm{mrad}$ for each pixel along the full FUV (EUV) slit.

[12] The calibration and instrument features of the UVIS are described in Esposito et al. [2004] and most recently by Stevens et al. [2011]. The primary standards used for determining the UVIS absolute radiometric sensitivity prior to launch were photodiodes made available by the National Institute for Standards and Technology (NIST). These standards were used to calibrate the UVIS. Measurements of the star Alpha Virginis (Spica) obtained during cruise (16-17 January 1999) agreed with previous stellar spectra to within $10 \%$ for both EUV and FUV channels, confirming the UVIS laboratory results. Periodic stellar calibrations (every 100 days on average since 2006) obtained during cruise and in orbit track changes in UVIS sensitivity. From these stellar observations we assess the UVIS calibration uncertainty to be $15 \%$ or better for all wavelengths.

[13] From 19 April 2009 through 28 January 2010, the UVIS instrument obtained $71 \mathrm{EUV} / \mathrm{FUV}$ spectral data pairs of Titan observations. Many of the observations occurred on the same Titan passes, numbered T53 through T66. The observation interruptions occurred because of sequenced pointing changes on Titan. During this period, the full 1024 wavelength channels $\times 60$ spatial channels were employed for these observations without any spectral or spatial binning or windowing that would limit the information content of the observations. These data files, including geometry are labeled by observation number in this work, with the first EUV/FUV pair on 19 April, 2009 through the last observation on 28 January 2010 . We call these records of data and geometry "data cubes." We abbreviate these observations without parenthesis as F0, F1, etc., through F71. Each observation of the 2009/2010 set lasts at least 12 min with files F1 and F2, the smallest set consisting of 3 records of $4 \mathrm{~min}$ each and the largest including 31 records F67 with a maximum Titan observation time of $124 \mathrm{~min}$ per UV spectral channel. The UVIS instrument is angularly scanning between multiples of 4-min stops, nominally, at an angular slew rate to move the UVIS bore sight from the exobase observations of Titan on one side of the body to the other side (total distance in plane of sky of 6000-8000 km). The only exception occurred for F14 described by Stevens et al. [2011] which is a high S/N "stare" observation. The spacecraft motion is compensated by an equal and opposite spacecraft slew. Similarly the 7 May 2009 eclipse observation was a 2-hr "stare" at a Titan disk and limb within Saturn's shadow. In summary, the nominal angular slews across Titan occur in segments of multiples of four minutes so the start-stop times coincide with multiples of 4-min dwells for ISS imaging.

[14] We show an over plot of the relevant major geometry information (mean (averaged over records and pixels)] SZA (top) and mean tangent ray height distance - MRH (bottom)) in Figure 2 with the same color coding for each point to be used in identifying the chronological order of the 71 observational UVIS data cubes. The data-cubes are a three dimensional UVIS data file for each observation: spatial pixel, spectral pixel, and record number, the latter represents time. The color coding used in Figure 2 is black for F0, the first observation and red for F70, the last observation, with each record four minutes in length in pixel integration time. Fortunately the limb airglow observations analyzed here occurred successively in seven distinct time-ordered geometrical groups during 2009, labeled: A (T53), B (T54), C (T55), D (T56), E (T62), F (T62), G (T62, T63) in Figure 2. We identify in Figure 2 (top) the SZA range for the three types of limb observations: bright limb $\left(0-90^{\circ}\right)$, twilight limb $\left(90-120^{\circ}\right)$ and dark limb $\left(120-180^{\circ}\right)$. There is also a critical MRH window exhibited in Figure 2 (bottom) from 500 to $1400 \mathrm{~km}$ for observing the limb airglow. This is the altitude range for UV airglow generation for the entire breadth of the ionosphere: both solar photoelectron excited and magnetosphere plasma impacted. The Titan atmosphere has an optical depth of much greater than unity for the entire UVIS FUV and EUV spectral range for MRH altitudes below $350 \mathrm{~km}$, implying the UVIS cannot observe the surface of Titan.

[15] We display an overview plot of the average airglow limb spectrum, averaged over all records, altitudes and SZA of each of the seven types of EUV and FUV limb spectra identified from the observational group of 71 from 2009 in Figures $3 \mathrm{a}$ and $3 \mathrm{~b}$. We also include an eighth limb observation displaying the 22 June 2009 "stare" observation (labeled F14), the brightest daylight airglow to date (discussed in Paper 3). Many of these 71 limb observations also involved disk observations that we do not consider in detail. The $15 \%$ uncertainty in absolute sensitivity calibration plus statistical $\mathrm{S} / \mathrm{N}$ uncertainty leads to approximately a $30 \%$ root-sumsquare uncertainty in the spectral intensities [Stevens et al., 2011]. We will consider, in detail, one distinct airglow 

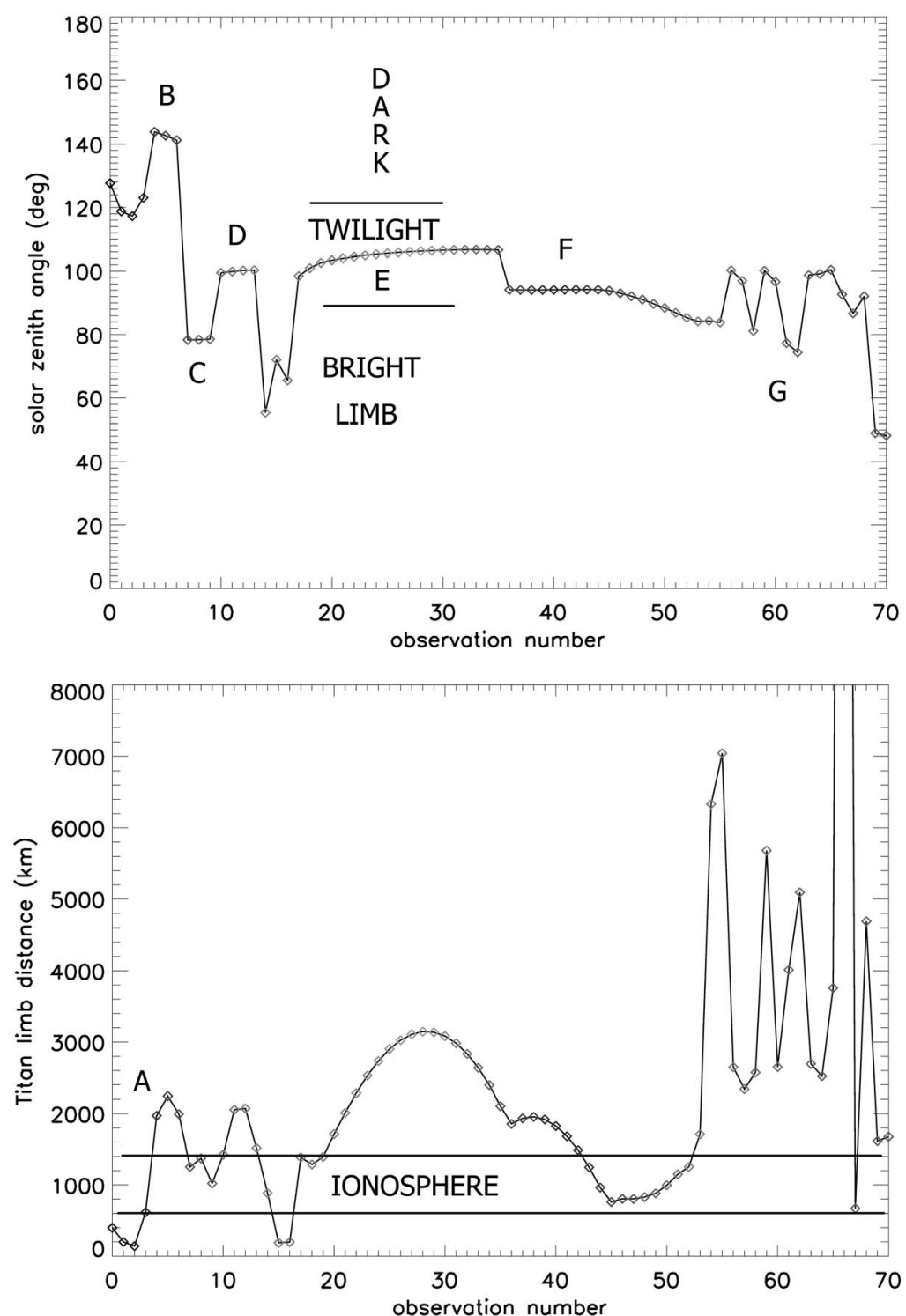

Figure 2. Over-plot of the major geometric parameters for the Titan limb observations with color coding from 0 (black) to 70 (red) according to observation number. These parameters are: (top) the mean SZA of the weighted mean pixel SZA for all the records of the observation; and (bottom) the mean Titan limb distance or MRH over all records of the observation. Figure 2 (top) shows the SZA regions for observing bright limb, twilight limb and dark limb observations. The seven different type of observations (labeled A,B,C,D,E,F, and G) with different geometric descriptions are identified in Table 1 by Titan flyby encounter number (column 1) and described (column 9).

observation from each of the seven groups, as identified by observation number in Figures $3 \mathrm{a}$ and $3 \mathrm{~b}$ and Table 1 . In addition, we consider the eclipse observation from 7May09 [West et al., 2011, 2012] and the comprehensive set of 25March08 observations of the two limb crossings (bright and twilight) and the two disk observations (bright and twilight).

[16] We show the UVIS slit field-of-view (FOV) upon or near Titan at the middle of record 0 for each of the eight limb (and disk) observations in Figure 4. The geometric parameters from Figure 4 are listed in Table 1 and include the Titan flyby number, spacecraft distance to Titan center, the average MRH over the range of limb distances included in the limb spectrum of the observation. The Table also includes the TPA and the mean SZA for the limb observation. The last column describes the limb observation description of Titan shown in Figures $3 \mathrm{a}$ and $3 \mathrm{~b}$ for each of the observation types. For example, F48 that is initiated on 2009 DOY $28515^{\mathrm{h}} 05^{\prime}$ is very bright in both the EUV and FUV with the slit spatial pixels on either the bright limb or bright disk at near $10^{\circ}$ phase angle. F14 was the brightest afternoon limb observation and the slit center was maintained in position on the airglow layer near $800 \mathrm{~km}$ for the central spatial pixels MRH altitude for one hour. The brightest pixels were centered at $1000 \mathrm{~km}$ altitude. Most of the observations to be discussed 

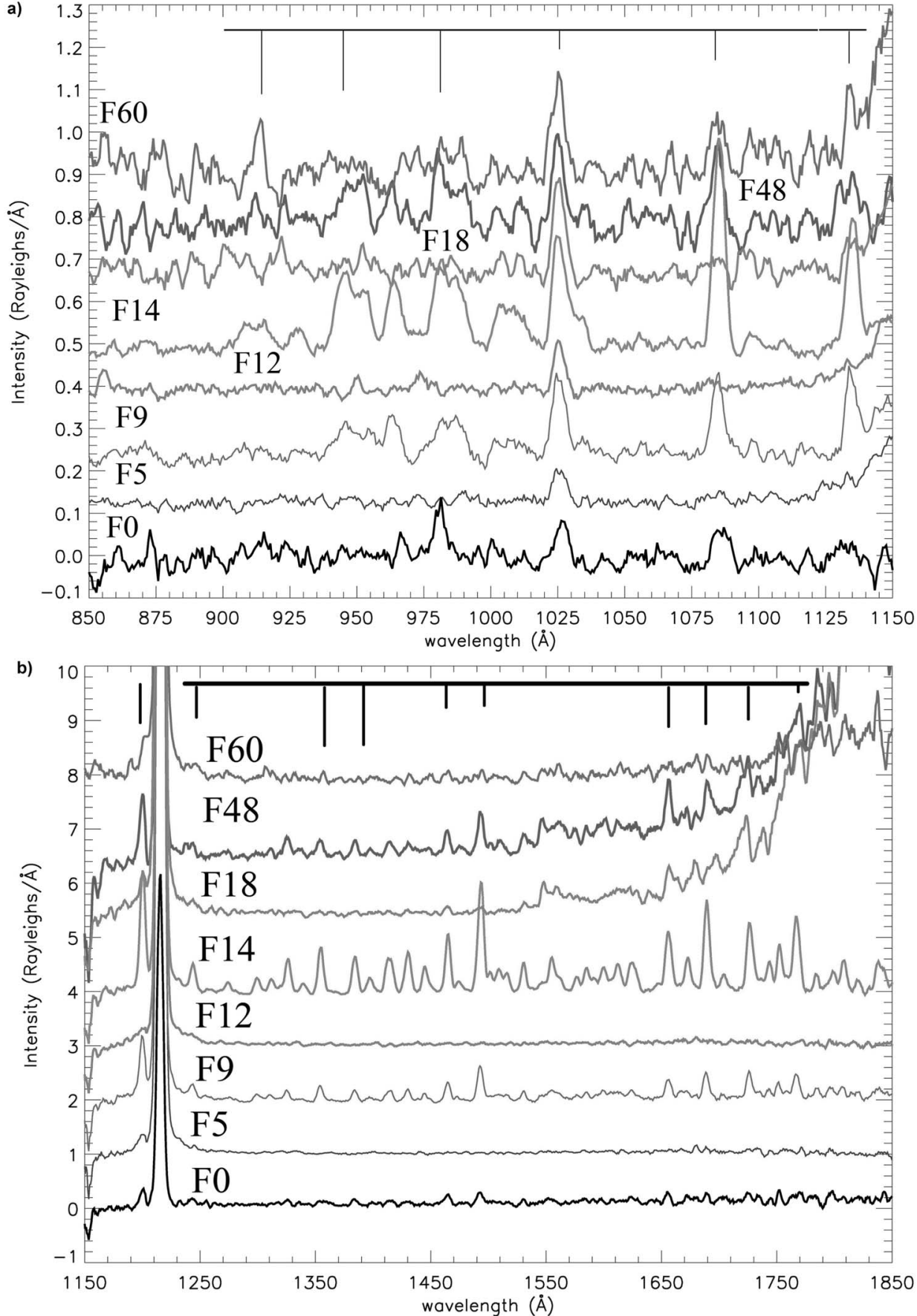

Figure 3. (a) EUV plots of individual limb spectra from regions A, B, C, D, E, F, and G of Figure 2 and described in first and last columns of Table 1. The observation number is given above each of the individual spectra. The color in the spectral plot corresponds to the same color as the corresponding observation number in Figure 2. The wavelength position of strong spectral emissions are indicated at the top of the plot. For example, the positions of NII $(919 \AA), \mathrm{N}_{2} c_{4}^{\prime \prime} \sum_{u}^{+}\left(\mathrm{v}^{\prime}=4,3\right) \rightarrow \mathrm{X}\left(\mathrm{v}^{\prime \prime}=3,2\right)$ at $945 \AA, \mathrm{N}$ I $(953 \AA)$, $c_{4}^{\prime}{ }^{1} \sum_{u}^{+}\left(\mathrm{v}^{\prime}=0\right) \rightarrow \mathrm{X}\left(\mathrm{v}^{\prime \prime}=1\right)$ at $980 \AA, \mathrm{H} \mathrm{Ly} \beta$ at $1025 \AA, \mathrm{N}$ II $(1085 \AA), \mathrm{N}$ I $1134 \AA$ are indicated and are found to be especially strong on F14, 22 June 2009. (b) FUV plots of individual limb spectra from regions A, B, C, D, E, F, and $G$ of Figure 3a and identified by Titan Flyby encounter number in column 1 of Table 1. (b) The observation number is given above each of the individual spectra. The color of the spectral plot corresponds to the same color as the corresponding observation number in Figure 2. The wavelength positions of strong spectral emissions are indicated at the top of the plot. For example, the wavelength positions of the strong vibrational bands of the Lyman-Birge-Hopfield band system $\left(\mathrm{a}^{1} \Pi_{\mathrm{g}}\left(\mathrm{v}^{\prime}=3,2,1\right) \rightarrow \mathrm{X}^{1} \sum_{g}^{+}\left(\mathrm{v}^{\prime \prime}=0,1,2\right)\right.$ of $\mathrm{N}_{2}, \mathrm{NI}$ $(1200,1243,1493)$ multiplets from solar photodissociative excitation of $\mathrm{N}_{2}$ and several vibrational bands from the $\mathrm{N}_{2} \mathrm{VK}$ system $\left(\mathrm{A}^{3} \sum_{\mathrm{u}}^{+}-\mathrm{X}^{1} \sum_{\mathrm{g}}^{+}\right.$system) longward of $1550 \AA$ are indicated. 


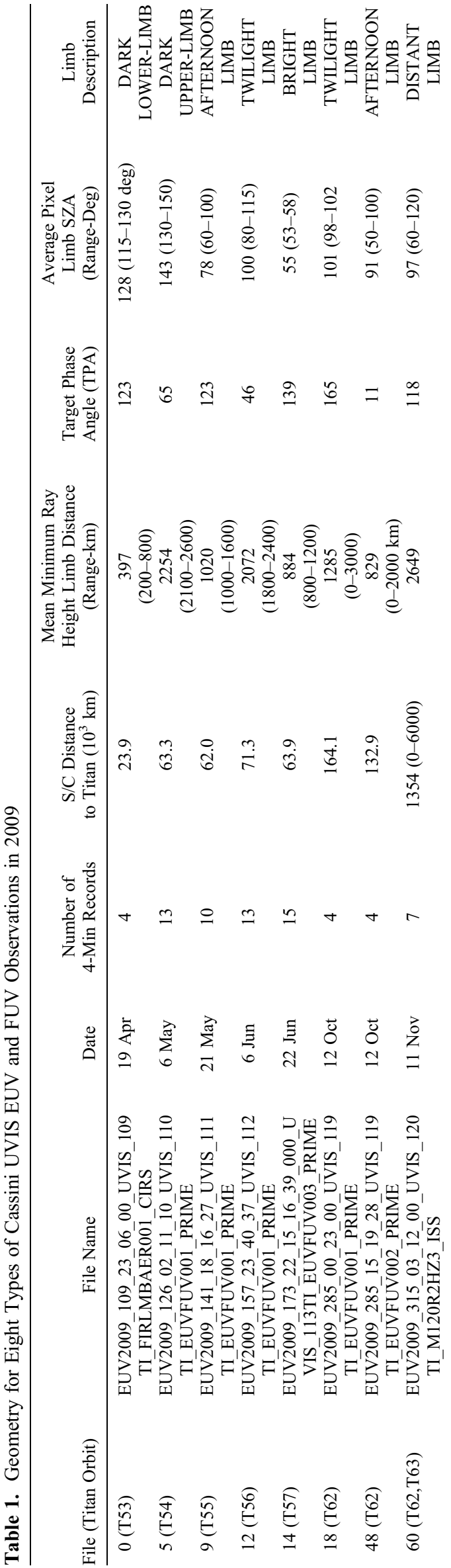

from the 2009 data set were at Titan-spacecraft distance of $170,000 \mathrm{~km}$ or less that represents an altitude resolution of $340 \mathrm{~km}$ or less in the EUV and $255 \mathrm{~km}$ or less in the FUV in the vicinity of the airglow layer, except for F60 which is a distant limb observation at $1.4 \times 10^{6} \mathrm{~km}$ from Titan. This distant observation is a measurement frequently encountered during the Cassini mission. We will see that observations over $5 \times 10^{5} \mathrm{~km}$ are too distant to yield meaningful limb observations due to the large range of SZA and MRH included on the spectral pixels and the simultaneous inclusion of both (bright, dark) disk and limb emissions on certain pixels. All of the other observations from Figures $3 \mathrm{a}$ and $3 \mathrm{~b}$ show limb spectra from either the bright or twilight or dark limb. The question remains what is the diurnal intensity variation over a period of a year for the brightest EUV and FUV features.

[17] We begin the discussion by noting that five of the spectra in Figures $3 \mathrm{a}$ and $3 \mathrm{~b}$ indicate both molecular $\mathrm{N}_{2}$ and atomic $\mathrm{N}$ emission features in both EUV and FUV. These observations are F0, F9, F14, F48 and F60. The EUV airglow emission spectra consistently exhibit the strong interplanetary (IP) and Titan H Ly $\beta$ atomic line, $c_{4}^{\prime}[0,1] 980 \AA$ molecular band intensity of $\mathrm{N}_{2}$ and N II $1085 \AA$ multiplet. The FUV features found in the five-spectra during the same observations occur with $\mathrm{N}_{2}$ molecular emissions for $\mathrm{LBH}$ and VK bands and the usual set of $\mathrm{N}$ I atomic multiplets (Papers 2 and 3 ). These features always seem to occur for solar photoexcitation for SZA $<120^{\circ}$ and MRH near $1000 \mathrm{~km}(800$ $1200 \mathrm{~km}$ ) and all Titan latitudes. The EUV emissions are strongly absorbed by $\mathrm{CH}_{4}$ for all Titan limb observations for MRH altitudes less than $800 \mathrm{~km}$. The UVIS observations depend on the FUV channel to remain optically thin below $800 \mathrm{~km}$ MRH until $350 \mathrm{~km}$ MRH over the wavelength range $1400-1900 \AA$ to allow tracking of low altitude limb airglow emissions. Magnetosphere plasma impact excitation probably occurs for F0 on the night side to account for EUV and FUV limb emissions and will be discussed in another section. We observe in Figures $3 \mathrm{a}$ and $3 \mathrm{~b}$ that there are two spectra without detectable Titan-based EUV and FUV emissions. These observations are F5, and F12. F5 and F12 are dark for two reasons: 1) minimum ray heights of $2100-2600 \mathrm{~km}$ for F5 and 1800-2400 km for F12, both MRH sets lying above the solar and magnetosphere excited ionosphere emission altitudes of $500-1200 \mathrm{~km}$ and 2) a mean dark-side SZA of $143^{\circ}$ for $\mathrm{F} 5$ and $100^{\circ}$ for $\mathrm{F} 12$.

[18] The F18 limb spectrum is weak in the EUV (no indication of the $\mathrm{N}_{2} c_{4}^{\prime}{ }^{1} \sum_{y}^{+}(0,1)$ band at $980 \AA$ or N II multiplet $\left(\mathrm{g}^{3} \mathrm{P}_{-}{ }^{3} \mathrm{P}^{\mathrm{o}}\right)$ near $\left.1085 \AA\right)$. The FUV indicates particle scattering only, without discernible $\mathrm{N}_{2}$ molecular bands or $\mathrm{N}$ I multiplets, despite containing an MRH in the ionosphere (see Figure 2). IP Ly $\beta$ (see West et al. [2012] for a discussion) is consistently present in all of the spectra in Figure 3a. This absence of nitrogen emission features on the limb is unusual because the FUV limb spectrum of F18 indicates particle scattering and shows that the topside ionosphere is illuminated by the solar FUV. The F18 limb pixels in the limb spectra in Figures $3 \mathrm{a}$ and $3 \mathrm{~b}$ cover the range from 0 to $3000 \mathrm{~km}$ for all four records while the SZA is at $101^{\circ}$ implying that the upper atmosphere at $\sim 1000 \mathrm{~km}$ is in daylight. This conclusion is verified in that the FUV limb spectrum indicates a strong Rayleigh scattered component. The 

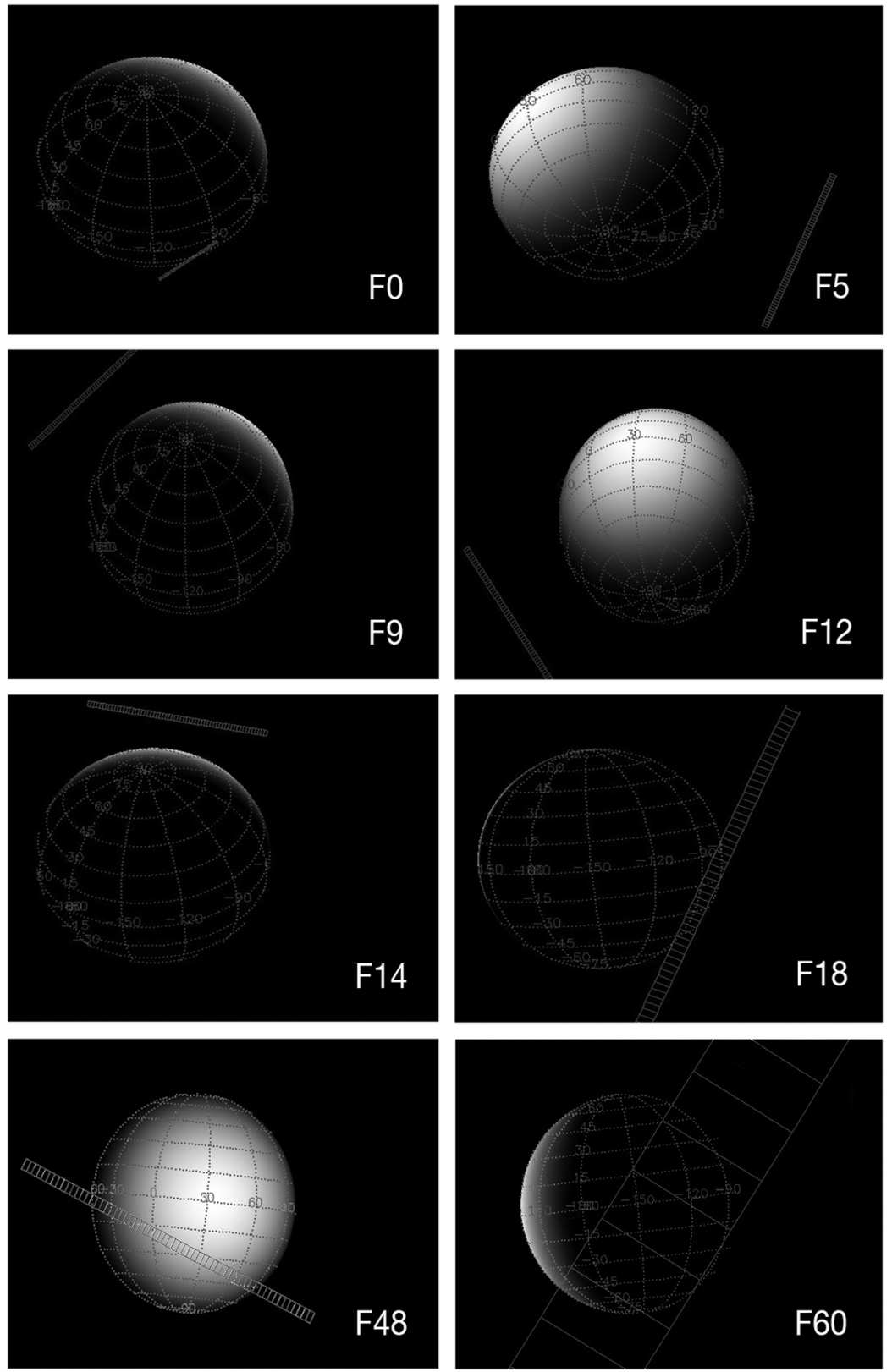

Figure 4. Geometric plots of each of the Titan limb and disk observations in 2009 by the EUV slit $(2 \mathrm{mrad} \times 60 \mathrm{mrad})$ demarking the EUV and FUV UVIS spectral observations in Figures $3 \mathrm{a}$ and $3 \mathrm{~b}$ limb at time of Record 0 . The geometric parameters for the observation are given in the data cubes in Table 1.

VK bands (some are indicated) and weak NI FUV multiplets are observed on the twilight limb (see below) in the FUV.

[19] To continue the analysis, we note in Figure $3 \mathrm{~b}$ that the FUV limb observations sometimes contain a longwavelength contribution from particle scattering and solar reflection, e.g., F18, F48, and F60. The bright limb and twilight limb observations (pixels with SZA $<120^{\circ}$ ) that have a portion of the $\sim 0.1^{\circ}$ field of view (FOV) minimum ray height that passes through the lower thermosphere - layers 2 and 3 $(300-600 \mathrm{~km})$ in Figure 1 during an observation will contain this contribution as our published UVIS dayglow limb spectra as a function of altitude in Sittler et al. [2010a, Figure 16.24] shows. If we consider the solar generated top side airglow layer $400 \mathrm{~km}$ wide [800-1200 km] (Paper 3) than a UVIS look direction toward the MRH of $800 \mathrm{~km}$ with a FOV of $1.5 \mathrm{mR}$ will include the lower thermosphere of reflecting haze at $500 \mathrm{~km}$ for a (closest approach) C/A distance of $150,000 \mathrm{~km}$. We see why F18 and F60 include this twilight component as the $\mathrm{C} / \mathrm{A}$ distance is more than $150,000 \mathrm{~km}$. We use solar reflection as an important discriminator for separating twilight glow and nightglow limb observations.

[20] We consider the analysis of another important aspect of the 71 observations in 2009 by comparing intensities of the strongest EUV and FUV features over the course of the year (2009 to early 2010) at the limb regions from Figure 2: the bright limb $\left(\mathrm{SZA}<90^{\circ}\right)$, the twilight $\operatorname{limb}\left(90^{\circ}<\mathrm{SZA}<120\right.$, and the dark limb $\left(\mathrm{SZA}>120^{\circ}\right)$. It is important to compare simultaneously the FUV intensities of two strong features to 

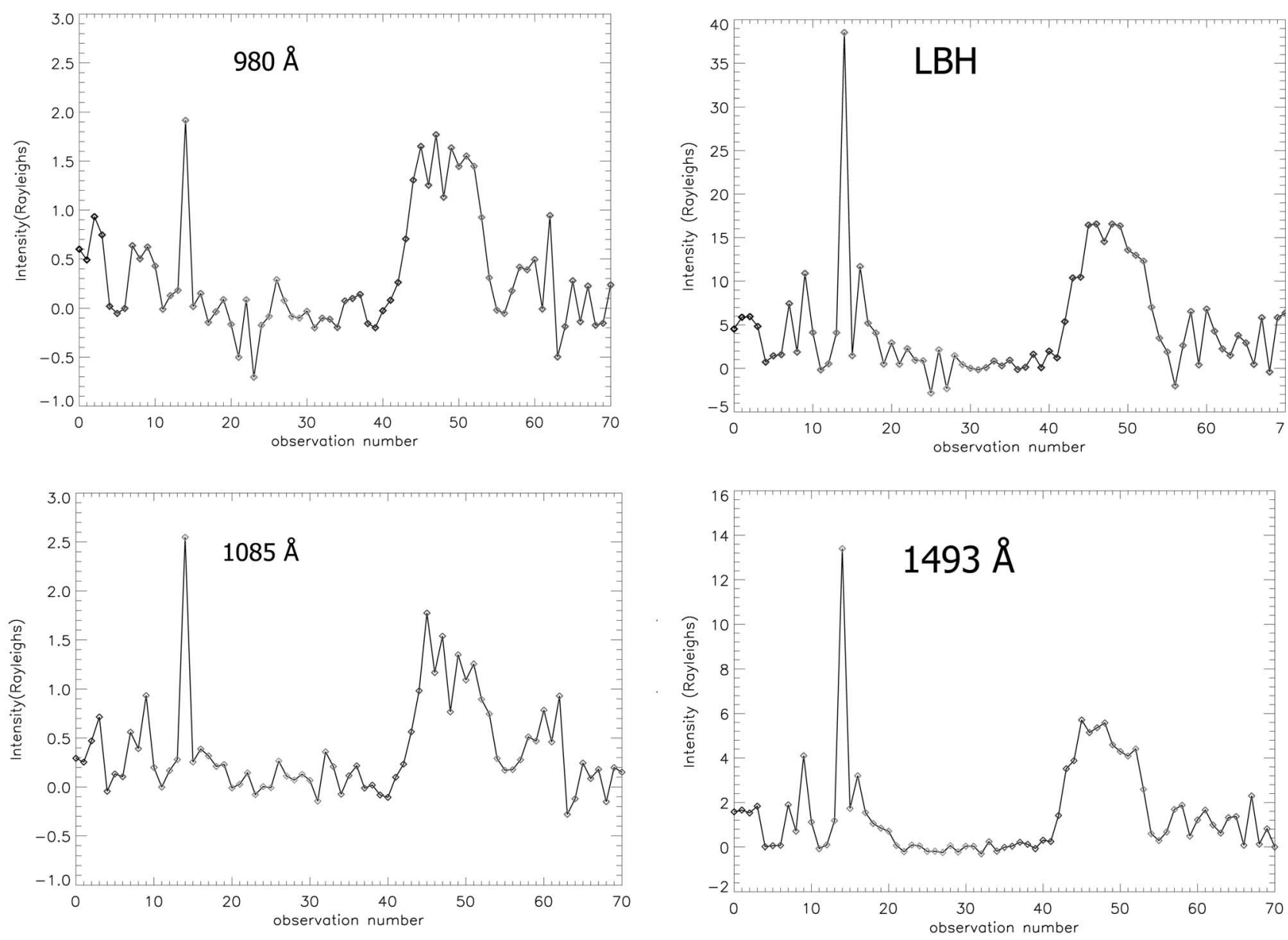

Figure 5. Mean limb intensities of EUV N II multiplet $\left(\mathrm{g}^{3} \mathrm{P}-{ }^{3} \mathrm{P}^{\mathrm{o}}\right)$ near $1085 \AA$ and the $c_{4}^{\prime}(0,1)$ band at $980 \AA$ and limb intensities of FUV N I multiplet $\left(g^{2} \mathrm{D}^{\circ}-{ }^{2} \mathrm{P}\right)$ at $1493 \AA$ and the LBH bands from 1300 to $1400 \AA$ for the 71 observations of 2009 and early 2010 with the same color scheme for each observation, as defined in Figure 2. Small negative intensity numbers occur for weak emissions of some observations in the subtraction of signal minus background.

correlate emissions also generated the same way as the EUV by photoelectrons, magnetosphere plasma and PDI of $\mathrm{N}_{2}$. We have shown in Papers 1 and 3 that the two brightest EUV emission features are the $\mathrm{N}$ II multiplet $\left(\mathrm{g}^{3} \mathrm{P}_{-}{ }^{3} \mathrm{P}^{\mathrm{o}}\right)$ near $1085 \AA$ and the $c_{4}^{\prime}(0,1)$ band at $980 \AA$, both with peak limb and bright disk intensities of near 2-3 R. We have shown, in Paper 2, that two of the brightest FUV emission features are the N I multiplet $\left(\mathrm{g}^{2} \mathrm{D}^{\mathrm{o}}{ }_{-}{ }^{2} \mathrm{P}\right)$ near $1493 \AA$ and the $\mathrm{LBH}$ bands (a ${ }^{1} \Pi_{\mathrm{g}}-\mathrm{X}^{1} \sum_{g}^{+}$band system) with strongest vibrational features from 1300 to $1400 \AA$ [Ajello et al., 2010, 2011a, 2011b; Ajello and Shemansky, 1985], both with peak limb and bright disk intensities of near $5 \mathrm{R}$ and near $100 \mathrm{R}$ (respectively, Papers 2 and 3). We plot for the limb the $c_{4}^{\prime}(0,1) 980 \AA$ molecular band intensity and LBH band intensity from 1295 to $1480 \AA$, N II $1085 \AA$ and N I $1493 \AA$ in Figure 5. We choose N I $1493 \AA$, which is only secondary to NI $1200 \AA$ in integrated intensity. The NI $1200 \AA$ multiplet is slightly blended with Ly $\alpha$ at $1216 \AA$. We track the integrated N I, II feature intensities for the year and these features track each other nearly identically.
[21] We also show the same data again in a similar way using tabular form. From the set of 71 UVIS bright limb observations identified by data-cube name, we summarize in Table 2 the mean values of the limb intensities of the $c_{4}^{\prime}(0,1)$ $980 \AA$ molecular band intensity, the LBH band system intensity from 1250 to $2500 \AA$ N II $1085 \AA$ and N I $1493 \AA$ multiplet intensities. We distinguish the observations by the call-outs shown in Table 2 with " $d$ " for bright limb dayglow, "t" for twilight limb and " $n$ " for limb nightglow with an additional indication of " $h$ " for a line-of-sight MRH that is high above ionosphere $(>1500 \mathrm{~km})$ and "far" for a spacecraft to Titan distance of greater than $1 \times 10^{6} \mathrm{~km}$. The table is useful for separately following daylight (d), twilight (t) or nightglow (n) intensities of limb observations within the ionosphere. For example, the brightest limb observation (F14) for both atomic features occurred on 22 June 2009 with a spacecraft distance to Titan of $64,000 \mathrm{~km}$ and a limb "stare" $\mathrm{MRH}$ at $800 \mathrm{~km}$ (for middle pixels $30-35$ ), SZA of $53^{\circ}$ and TPA of $139^{\circ}$. Observations of the atomic features in daylight significantly above $800 \mathrm{~km} \mathrm{MRH} \mathrm{(observations} \mathrm{7,} \mathrm{8,} 9$ in 
Table 2. Limb Intensities of Brightest EUV and FUV Features

\begin{tabular}{|c|c|c|c|c|c|c|}
\hline \multirow[b]{2}{*}{ Observation } & \multirow[b]{2}{*}{ UVIS FUV File Name } & \multicolumn{4}{|c|}{ Limb Intensity (Rayleighs) Airglow ${ }^{a}$} & \multirow[b]{2}{*}{ Notes $^{b}$} \\
\hline & & $980 \AA$ & $1085 \AA$ & LBH & $1493 \AA$ & \\
\hline 0 & FUV2009_109_23_06_00_UVIS_109TI_FIRLMBAER001_CIRS & 0.60 & 0.29 & 12.2 & 1.58 & $\mathrm{n}$ \\
\hline 1 & FUV2009_109_23_27_39_UVIS_109TI_FIRLMBAER001_CIRS & 0.49 & 0.25 & 15.9 & 1.66 & $\mathrm{n}$ \\
\hline 2 & FUV2009_109_23_42_48_UVIS_109TI_FIRLMBAER001_CIRS & 0.93 & 0.47 & 16.1 & 1.53 & $\mathrm{n}$ \\
\hline 3 & FUV2009 $110-011^{-} 34^{-}$UVIS $^{-}$109TI FIRLMBAER002- CIRS & 0.75 & 0.72 & 13.0 & 1.83 & $\mathrm{n}$ \\
\hline 4 & FUV2009_126_01_18_21_UVIS_110TI_EUVFUV001_PRIMTE & 0.02 & 0.04 & 1.96 & 0.01 & $\mathrm{nh}$ \\
\hline 5 & FUV2009_126_02_11_10_UVIS_110TI_EUVFUV001_PRIME & -0.05 & 0.13 & 3.92 & 0.06 & $\mathrm{nh}$ \\
\hline 6 & FUV2009_126_03_03_59_UVIS_110TIEEUVFUV001_PRIME & 0.0 & 0.10 & 4.28 & 0.07 & nh \\
\hline 7 & FUV2009_141_16_38_15_UVIS_111TI_EUVFUV001_PRIME & 0.64 & 0.56 & 20.1 & 1.89 & $\mathrm{~d}$ \\
\hline 8 & FUV2009_141_17_27_21_UVIS_111TI_EUVFUV001_PRIME & 0.50 & 0.39 & 5.05 & 0.71 & $\mathrm{~d}$ \\
\hline 9 & FUV2009_141_18_16_27_UVIS_111TIEEUVFUV001_PRIME & 0.62 & 0.93 & 29.4 & 4.10 & $\mathrm{~d}$ \\
\hline 10 & FUV2009_157_21_48_36_UVIS_112TI_EUVFUV001_PRIME & 0.43 & 0.20 & 11.1 & 1.12 & $\mathrm{~d}$ \\
\hline 11 & FUV2009 $157^{-} 22^{-} 44^{-} 36^{-}$UVIS $^{-} 112 \mathrm{TI}^{-}$EUVFUV001-PRIME & 0.01 & 0.00 & -0.46 & -0.07 & th \\
\hline 12 & FUV2009_157_23_40_37_UVIS_112TI_EUVFUV001_PRIME & 0.13 & 0.17 & 1.44 & 0.09 & th \\
\hline 13 & FUV2009_158_00_36_38_UVIS_112TI_EUVFUV001_PRIME & 0.18 & 0.28 & 11.05 & 1.18 & $\mathrm{t}$ \\
\hline 14 & FUV2009_173_15_16_39_UVIS_113TI_EUVFUV003_PRIME & 1.92 & 2.55 & 104.1 & 13.4 & $\mathrm{~d}$ \\
\hline 15 & FUV2009_189_12_55_05_UVIS_114TIEEUVFUV002_PRIME & 0.012 & 0.26 & 3.92 & 1.73 & $\mathrm{~d}$ \\
\hline 16 & FUV2009_189_13_47_22_UVIS_114TIEEUVFUV002_PRIME & 0.15 & 0.39 & 31.53 & 3.20 & $\mathrm{~d}$ \\
\hline 17 & FUV2009 $285^{-} 00^{-} 04^{-} 00^{-}$UVIS $^{-1} 19 \mathrm{TI}^{-}$EUVFUV001-PRIME & 0.14 & 0.32 & $14 . .1$ & 1.54 & $\mathrm{t}$ \\
\hline 18 & FUV2009_285_00_23_00_UVIS_119TIEEUVFUV001_PRIME & .04 & 0.21 & 11.0 & 1.05 & $\mathrm{t}$ \\
\hline 19 & FUV2009285_00_42_00_UVIS_119TIEEUVFUV001_PRIME & 0.09 & 0.23 & 1.36 & 0.85 & $\mathrm{t}$ \\
\hline 20 & FUV2009_285_01_01_00_UVIS_119TI_EUVFUV001_PRIME & 0.16 & -0.01 & 7.93 & 0.71 & $\mathrm{t}$ \\
\hline 21 & FUV2009_285_01_20_00_UVIS_119TI_EUVFUV001_PRIME & -0.50 & 0.03 & 1.30 & 0.06 & th \\
\hline 22 & FUV2009_285_01_39_00_UVIS_119TI_EUVFUV001_PRIME & 0.09 & 0.14 & 6.16 & -.20 & th \\
\hline 23 & FUV2009_285_01_58_00_UVIS_119TIEEUVFUV001_PRIME & -0.70 & -0.08 & 2.52 & 0.09 & th \\
\hline 24 & FUV2009_285_02_17_00_UVIS_119TI_EUVFUV001_PRIME & -0.17 & 0.00 & 2.39 & 0.05 & th \\
\hline 25 & FUV2009 $285^{-} 02^{-} 36^{-} 00^{-}$UVIS $^{-1} 19 \mathrm{TI}^{-}$EUVFUV001-PRIME & -0.08 & -0.01 & -7.65 & -0.19 & th \\
\hline 26 & FUV2009_285_02_55_00_UVIS_119TIEEUVFUV001_PRIME & 0.29 & .27 & 5.78 & -0.18 & th \\
\hline 27 & FUV2009_285_03_14_00_UVIS_119TI_EUVFUV001_PRIME & 0.08 & 0.11 & -6.29 & 0.24 & th \\
\hline 28 & FUV2009_285_03_33_00_UVIS_119TI_EUVFUV001_PRIME & -0.09 & 0.07 & 3.97 & 0.06 & th \\
\hline 29 & FUV2009_285_03_52_00_UVIS_119TI_EUVFUV001_PRIME & -0.10 & 0.13 & 1.19 & -0.22 & th \\
\hline 30 & FUV2009_285_04_11_00_UVIS_119TI_EUVFUV001_ & -0.03 & 0.07 & 0.04 & 0.04 & th \\
\hline 31 & FUV2009 $285^{-} 04^{-} 30-00$ UVIS $119 T^{-}$EUVFUV001-PRIME & -0.20 & -0.14 & -0.41 & 0.03 & th \\
\hline 32 & FUV2009_285_04_49_00_UVIS_119TI_EUVFUV001_PRIME & -0.10 & 0.36 & 0.28 & -0.30 & th \\
\hline 33 & FUV2009285_05_08_00_UVIS_119TIEUVFUV001_PRIME & -0.12 & -0.12 & 2.33 & 0.24 & th \\
\hline 34 & FUV2009_285_05_27_00_UVIS_119TI_EUVFUV001_PRIME & -0.20 & -0.07 & 0.80 & -0.18 & th \\
\hline 35 & FUV2009_285_05_46_00_UVIS_119TI_EUVFUV001_PRIME & 0.08 & 0.12 & 2.55 & -0.01 & th \\
\hline 36 & FUV2009_285_11_41_05_UVIS_119TI_EUVFUV002_PRIME & 0.10 & 0.22 & -0.33 & 0.0 & th \\
\hline 37 & FUV2009_285_11_59_17_UVIS_119TI_EUVFUV002_PRIME & 0.14 & -0.01 & 0.38 & 0.21 & th \\
\hline 38 & FUV2009_285_12_17_29_UVIS_119TI_EUVFUV002_PRIME & -0.16 & 0.02 & 4.41 & 0.12 & th \\
\hline 39 & FUV2009 $285^{-} 12^{-} 35^{-} 41^{-}$UVIS $^{-} 119 \mathrm{TI}^{-}$EUVFUV002- PRIME & -0.20 & -0.08 & 0.24 & -0.07 & th \\
\hline 40 & FUV2009_285_12_53_53_UVIS_119TI_EUVFUV002_PRIME & -0.03 & -0.11 & 5.36 & 0.30 & th \\
\hline 41 & FUV2009285_13_12_05_UVIS_119TIEUVFUV002_PRIME & 0.08 & 0.10 & 3.31 & 0.25 & th \\
\hline 42 & FUV2009_285_13_30_17_UVIS_119TI_EUVFUV002_PRIME & 0.26 & 0.23 & 14.5 & 1.41 & $\mathrm{~d}$ \\
\hline 43 & FUV2009_285_13_48_28_UVIS_119TI_EUVFUV002_PRIME & 0.70 & 0.56 & 28.0 & 3.52 & $\mathrm{~d}$ \\
\hline 44 & FUV2009_285_14_06_40_UVIS_119TIEEUVFUV002_PRIME & 1.31 & 0.98 & 28.2 & 3.88 & $\mathrm{~d}$ \\
\hline 45 & FUV2009_285_14_24_52_UVIS_119TIEEUVFUV002_PRIME & 1.65 & 1.77 & 44.4 & 5.71 & $\mathrm{~d}$ \\
\hline 46 & FUV2009_285_14_43_04_UVIS_119TIEEUVFUV002_PRIME & 1.25 & 1.17 & 44.7 & 5.14 & $\mathrm{~d}$ \\
\hline 47 & FUV2009285_15_01_16_UVIS_119TIEUVFUV002_PRIME & 1.77 & 1.54 & 39.3 & 5.36 & $\mathrm{~d}$ \\
\hline 48 & FUV2009_285_15_19_28_UVIS_119TI_EUVFUV002_PRIME & 1.13 & 0.77 & 44.7 & 5.58 & $\mathrm{~d}$ \\
\hline 49 & FUV2009285_15_37_40_UVIS_119TIEUVFUV002_PRIME & 1.64 & 1.35 & 44.2 & 4.59 & $\mathrm{~d}$ \\
\hline 50 & FUV2009_285_15_55_52_UVIS_119TI_EUVFUV002_PRIME & 1.45 & 1.09 & 36.6 & 4.29 & $\mathrm{~d}$ \\
\hline 51 & FUV2009_285_16_14_04_UVIS_119TI_EUVFUV002_PRIME & 1.55 & 1.26 & 35.0 & 4.09 & $\mathrm{~d}$ \\
\hline 52 & FUV2009_285_16_32_16_UVIS_119TIEEUVFUV002_PRIME & 1.45 & 0.90 & 33.2 & 4.41 & $\mathrm{~d}$ \\
\hline 53 & FUV2009_285_16_50_29_UVIS_119TI_EUVFUV002_PRIME & 0.93 & 0.75 & 19.0 & 2.59 & $\mathrm{~d}$ \\
\hline 54 & FUV2009_289_15_17_59_UVIS_119TI_M90R3CLD289_ISS & 0.31 & 0.29 & 9.43 & 0.60 & far \\
\hline 55 & FUV2009_290_04_24_59_UVIS_119TI_M90R3CLD290_ISS & -0.02 & 0.17 & 5.12 & 0.29 & far \\
\hline 56 & FUV2009_299_14_09_59_UVIS_120TI_M150R2HZ299_ISS & -0.05 & 0.18 & -5.41 & 0.68 & far \\
\hline 57 & FUV2009-303_03-42-29-UVIS_120TI_M90R2CLD303_ISS & 0.18 & 0.28 & 7.11 & 1.69 & th \\
\hline 58 & FUV2009_304_03_42_29_UVIS_120TI_M90R2CLD304_ISS & 0.42 & 0.51 & 17.7 & 1.87 & dh \\
\hline 59 & FUV2009_307_13_40_59_UVIS_120TI_M90R3CLD307_ISS & 0.39 & 0.47 & 1.10 & 0.41 & far \\
\hline 60 & FUV2009_315_03_12_00_UVIS_120TI_M120R2HZ315_ISS & 0.49 & 0.7 & 18.4 & 1.22 & th \\
\hline 61 & FUV2009_317_20_27_59_UVIS_121TI_M90R2CLD317_ISS & -0.01 & 0.46 & 11.5 & 1.66 & far \\
\hline 62 & FUV2009_319_02_56_59_UVIS_121TI_M90R3CLD319_ISS & 0.94 & 0.93 & 6.06 & 0.99 & far \\
\hline 63 & FUV2009_326_14_52_59_UVIS_121TI_M150R2HZ326_ISS & -0.50 & -0.28 & 4.05 & 0.62 & far \\
\hline 64 & FUV2009_331_19_44_59_UVIS_121TI_M90R2CLD331_ISS & -0.19 & 0.12 & 10.2 & 1.32 & far \\
\hline 65 & FUV2009_333_01_59_00_UVIS_121TI_M90R2CLD333_ISS & 0.28 & 0.24 & 7.91 & 1.37 & far \\
\hline 66 & FUV2009 $334 \quad 21 \quad 42 \quad 04$ UVIS 122TI ECLIPSE001 ISS & -0.14 & 0.09 & 1.25 & 0.07 & \\
\hline
\end{tabular}


Table 2. (continued)

\begin{tabular}{|c|c|c|c|c|c|c|}
\hline \multirow[b]{2}{*}{ Observation } & \multirow[b]{2}{*}{ UVIS FUV File Name } & \multicolumn{4}{|c|}{ Limb Intensity (Rayleighs) Airglow ${ }^{a}$} & \multirow[b]{2}{*}{ Notes $^{\mathrm{b}}$} \\
\hline & & $980 \AA$ & $1085 \AA$ & LBH & $1493 \AA$ & \\
\hline 67 & FUV2009_361_19_23_43_UVIS_123TI_FIRNADMAP001_CIRS & 0.23 & 0.18 & 15.7 & 2.30 & $\mathrm{t}$ \\
\hline 68 & FUV2009_361_21_34_10_UVIS_123TI_FIRNADMAP001_CIRS & -0.17 & 0.15 & -1.10 & -0.40 & th \\
\hline 69 & FUV2010 $028 \quad 18 \quad 04 \quad 24$ UVIS 125 TI EUVFUV001 PRIME & -0.15 & 0.19 & 15.8 & 0.82 & dh \\
\hline 70 & FUV2010_028_18_31_24_UVIS_125TI_EUVFUV001_PRIME & 0.24 & 0.15 & 17.2 & 0.00 & $\mathrm{dh}$ \\
\hline
\end{tabular}

${ }^{\mathrm{a}}$ Weak signals may produce a negative intensity result for (Signal + Noise) - Noise indicated in the Limb Intensity columns.

botes: d, day; t, twilight; $\mathrm{n}$, night; h indicates limb observation higher than $1500 \mathrm{~km}$ altitude MRH; far indicates limb observations with spacecraft distance to Titan further then $10^{6} \mathrm{~km}$.

Figure 2) or below $800 \mathrm{~km} \mathrm{MRH} \mathrm{(observations} \mathrm{15,} \mathrm{16)} \mathrm{are}$ weaker). Similar yearly correlations exist with the LBH band system intensity and the $c_{4}^{\prime}(0,1) 980 \AA$ molecular band intensity. The brightest nightglow observations occurred on F2 and F3 with a significantly greater emphasis on low altitude MRH observations of $350-800 \mathrm{~km}$ (to be discussed in section 4.0).

[22] The bright disk EUV observations, not shown in the figures of the $71 \mathrm{limb}$ observations (we show only limb at MRH $>0 \mathrm{~km}$ tangent altitude), occurs on many observations with strong brightness in all these features on DOY285 F45F52. The bright disk and limb EUV spectra of F45 - F52 are nearly as bright as F14 as shown in Figure 5. For example the brightness of $1085 \AA$ is 2.5 Ray on F14 and is 1.5 Ray on F48. The bright limb intensities occurring on DOY285 for spacecraft-Titan distances of $120-160 \mathrm{k} \mathrm{km}$ are at similar limb distances (MRH) of approximately $1000 \mathrm{~km}$ of 22 June 2009 for SZA of $80-90^{\circ}$ and a TPA of $10^{\circ}$ (fully illuminated disk). Thus the bright limb for DOY285 was observed at a near mean $80-90^{\circ}$ SZA. However, the distance to Titan was $132,900 \mathrm{~km}$ during F48 and the FOV of the FUV was $200 \mathrm{~km}$, sufficiently large enough with the spacecraft motion to observe the particle scattering present from spectral contributions below $600 \mathrm{~km}$, while the UVIS is pointed at the bright limb. The FWHM of the airglow layer intensities in $\mathrm{N}_{2}$ emissions for F48 is $200-300 \mathrm{~km}$. Only Titan flyby distance of $60,000 \mathrm{~km}$ or less, like F14, can spatially resolve the peak airglow intensity. Similarly F18 at 164,100 km with a FOV of $246 \mathrm{~km}$ and a SZA of $100^{\circ}$ presented a twilight limb airglow in Figure $3 \mathrm{~b}$ since the topside ionosphere was fully illuminated. The dark disk intensity measurements for 980 and $1085 \AA$ are measurable on only a few observations, especially F0 and F3, and are important in deciding on the general characteristics of the nightglow intensities compared to the dayglow intensities.

\section{Titan in Solar Eclipse}

[23] There was a solar eclipse observation within Saturn's shadow of Titan on 7 May 2009 by UVIS from a distance of $6.43 \times 10^{5} \mathrm{~km}$ for record 0 . The set of three eclipse observations from 2009 through 2010 are described in West et al. [2011, 2012]. In brief for the 7 May 2009 observation of the eclipse by UVIS there were 9-records $(0-8)$ with steady viewing of $160 \mathrm{~min}(2.675 \mathrm{~h})$ in Saturn's shadow. Each record was $1070 \mathrm{~s}\left(17.83^{\prime}\right)$ in duration) and contains 60 spectra. Out of that set of 9 records there were two types of individual spectra from each record: 1) the dark disk at exact center of the pixel (e.g., EUV and FUV spatial pixels 29 to 34 indicated dark disk and were summed for each of the 9 records for a total of
54 dark disk spectra and, 2) the dark limb (FUV spatial pixels 24 to 27 and 35 to 39: a total of 90 limb spectra) accounting for the remaining spatial pixels on the dark limb. The limb pixels range in MRH values from surface to $3000 \mathrm{~km}$. Most pixels outside of this set were eliminated as to be too distant from Titan to contribute to the limb spectra as each spatial pixel in the EUV accounts for a spatial resolution of $600 \mathrm{~km}$. The slit-Titan (surface) geometry is shown in Figure 6 for the record 5 (0-8 total records) at the mid-time on 7 May, $09^{\mathrm{h}} 57^{\prime}$. The mid- time of record 0 was 7 May, $08^{\mathrm{h}} 28^{\prime}$, and the midtime of record 8 was 7 May, $10^{\mathrm{h}} 50^{\prime}$. The slit was maintained in position identically for each record from 0 through 8 . The spacecraft distance was so great $(666,076 \mathrm{~km}$ for record 5) and the pointing was maintained adequately constant that only one additional pixel (each spatial pixel is $1 \mathrm{mrad}$ in angular FOV or $666 \mathrm{~km}$ in limb extent at Titan) needed to be included on the dark disk averaging with time. We included in the FUV average dark disk spectrum the average of pixel numbered 34, in the spectra from records 3, 5, 6 and 7 .

[24] The spectra of dark limb and dark disk are shown in Figure 7 for the EUV: 1) in red with a data boxcar smooth of 3 for adjacent points; and 2) an nsum mathematical value of 4 , which is used to obtain an average value of a single point for each group of 4 points. The EUV spectra of dark limb and dark disk are nearly identical with a similar intensity for each of the same 3 or 4 nitrogen features of peak intensities $\sim 60 \mathrm{mRay} / \AA \AA$ or about $0.2-0.5$ Ray intensity for the feature for a filled field of view (FOV). The distant FOV projection on the ionosphere may be under-filled compared to the nightglow extent, since the FWHM of the ionosphere layer is roughly $400 \mathrm{~km}$ [Kliore et al., 2008]. The observed features are: 1) $\mathrm{N}_{2} c_{4}^{\prime}{ }^{1} \sum_{u}^{+}(3,2 ; 4,3)$ at $\left.945 \AA ; 2\right)$ the $\left.\mathrm{N}_{2} c_{4}^{\prime}{ }^{1} \sum_{u}^{+}\right)(0,1)$ band at $980 \AA$; and 3$) \mathrm{N}$ II multiplet $\left(\mathrm{g}^{3} \mathrm{P}_{-}{ }^{3} \mathrm{P}^{\mathrm{o}}\right)$ near $1085 \AA$. In addition, we find the interplanetary $\mathrm{H} \mathrm{Ly} \beta$ emission with a peak intensity of near $70 \mathrm{mR} / \AA$ or an integrated intensity of near $0.46 \mathrm{R}$ on the limb and $0.30 \mathrm{R}$ on disk, indicating the disk was blocking the infinite column extent of IP $\mathrm{H} \mathrm{Ly} \beta$ with the bulk of the disk emission arising from two sources: a) scattering the interplanetary glow by the eclipsed disk of Titan [West et al., 2012] and b) proton charge exchange with $\mathrm{N}_{2}$ [Ajello et al., 2011b]. We calculate the directly observed IP $\mathrm{H} \mathrm{Ly} \beta$ contribution to the limb signal is about $0.16 \mathrm{R}$. The EUV nightglow features are about a factor of 5 less in intensity than the disk observations from the EUV dayglow (Paper 1) and about ten times less than limb dayglow observations (Paper 3) [West et al., 2012].

[25] We now proceed to a regression analysis of the EUV eclipse observations. The technique is explained in section 5.0, describing another set of limb observations occurring on 25 March 2008. For our eclipse analysis we choose to 


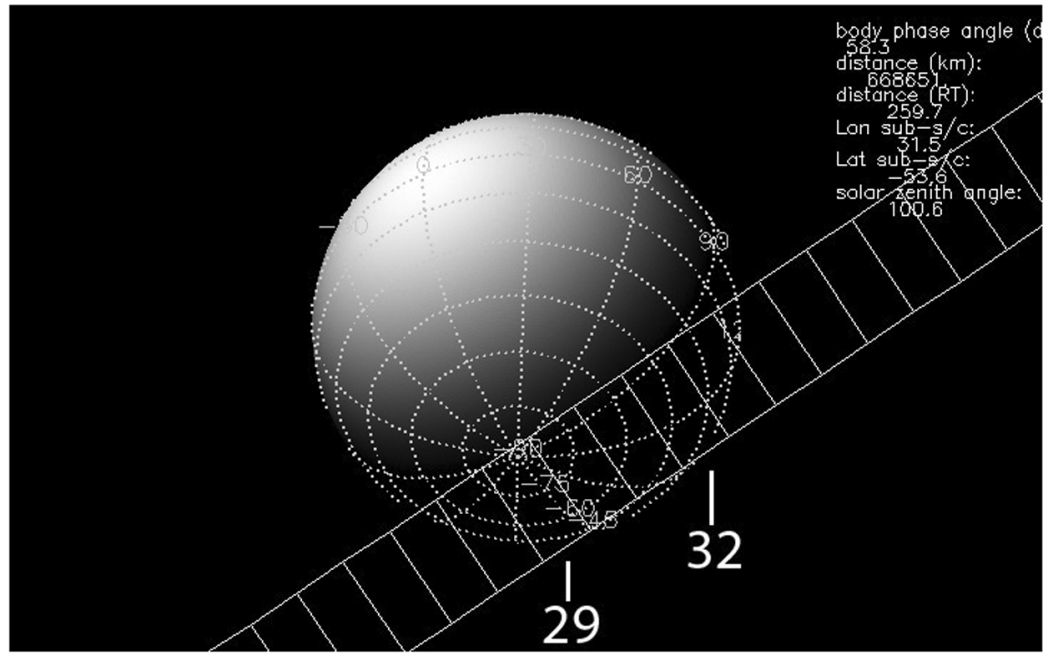

Figure 6. Geometric plot of each of the Titan limb observations during the eclipse as viewed by the Cassini UVIS slit at mid-time of Record 5 for 7 May $2009,09^{\mathrm{h}} 57^{\prime}$. The entire surface of Titan was in darkness. The UVIS slit is shown in the figure with the numbers 29 and 32 denoting the pixels, which are centered on the dark-disk from pixel 27 to 33. The mid-time of Record 0 was 7 May 2009, 08 $27^{\mathrm{h}}$, and the mid-time of record 8 was $10^{\mathrm{h}} 50^{\prime}$. The UVIS instrument was pointed at [RA,Dec] $=[200.3132,59.6999]$ in degrees and is viewing northward (upwards) from Titan sub-spacecraft latitude -53.59 degrees.

combine the dark limb and dark disk data set, since many pixels are a combination of limb and disk and to allow adequate average $\mathrm{S} / \mathrm{N}$ in the regression analysis. We show, in Figure 8 for the EUV, a composite regression optically thin model. The EUV spectrum is shown in Figure 8 from 900 to $1140 \AA$ with an integrated EUV intensity of $2.0 \mathrm{R}$ compared to a composite fit of $2.3 \mathrm{R}$. These intensities can be compared to a similar dayglow spectrum of 13 December 2004 analyzed by a regression analysis from 900 to $1140 \AA$ with a measured intensity of $16.6 \mathrm{R}$ and a modeled intensity of 17.5 R. For example the (Carroll-Yoshino) $\mathrm{CY}(0,1)$ band has a modeled eclipse intensity of $0.2 \mathrm{R}$. compared to $1.5 \mathrm{R}$ in the 13 December 2004 dayglow. We see approximately a factor of 8 difference in the EUV between dayglow and nightglow intensities.

[26] The dark limb and dark disk FUV spectra (not shown) are also interesting indicating three weak nitrogen features the $\mathrm{N}$ I multiplets $\left({ }^{4} \mathrm{~S}^{\mathrm{O}}{ }_{-}^{4} \mathrm{P}\right)$ at $1200(3.1 \mathrm{R}$ and $2.0 \mathrm{R})$ $\left({ }^{2} \mathrm{D}^{\mathrm{o}-}{ }_{-}^{2} \mathrm{D}\right)$ at $1243 \AA(0.94 \mathrm{R}$ and $0.52 \mathrm{R})$ : and $\left({ }^{2} \mathrm{D}^{\mathrm{o}}{ }_{-}{ }^{2} \mathrm{P}\right)$ at $1493 \AA$ (1.93 R and $0.55 \mathrm{R})$ on the disk and limb, respectively, and weak LBH band emissions: $(3,0)$ at $1356 \AA,(2,0)$ at $1383 \AA$ and $(1,1)$ at $1464 \AA$. The LBH bands, with sufficient $\mathrm{S} / \mathrm{N}$ to be seen on the limb only, have a peak intensity of about $100 \mathrm{mR} / \AA$ per feature or an integrated intensity about $0.5 \mathrm{R}$ per feature. These features represent about $5 \%$ of the band system and indicate $\sim 10 \mathrm{R}$ emission for the LBH band system [Ajello and Shemansky, 1985]. The H Ly $\alpha$ intensity is $122 \mathrm{R}$ on the limb and $35 \mathrm{R}$ on the disk, which leads to $87 \mathrm{R}$ signal intensity from IP H Ly $\alpha$ in the downwind direction of the interstellar wind at $20 \mathrm{R}_{\mathrm{s}}$. Additionally, we remind ourselves that the $\mathrm{H} \operatorname{Ly} \beta$ IP signal is $0.16 \mathrm{R}$. We find the ratio IP $\mathrm{H} \mathrm{Ly} \alpha / \mathrm{IP} \mathrm{H} \mathrm{Ly} \beta$ is 544 in the downwind direction [West et al., 2012]. In comparison the New Horizons observations of the IP emissions, observes a ratio of Ly $\alpha /$ Ly $\beta$ brightness of 600-800 (averaged over a particular 360-degree swath on the sky) [Gladstone et al.,
2012]. The intensity of H Ly $\alpha$ at $20 \mathrm{R}_{\mathrm{s}}$ in the UVIS look direction shown in Figure 6 for 27 May 2009 has negligible contribution from the magnetospheric distribution of $\mathrm{H}$ near Saturn [Melin et al., 2009].

[27] We show in Figure 9 the regression model of the FUV eclipse spectrum from $1175 \AA$ to $1775 \AA$ with combined limb and dark disk pixels for $\mathrm{S} / \mathrm{N}$ considerations as we performed for the EUV. The main difference between this eclipse FUV regression model and the dayglow FUV regression model lays in the fact that a solar scattering vector is not needed as the spectrum is flat and zero-level at high wavelength to $1900 \AA$ except for very weak VK bands. The entire sphere of the moon lies in darkness. The model for the LBH bands over predicts the $\mathrm{LBH}$ emission rate at $19.8 \mathrm{R}$ compared to an analysis of the limb spectrum, yielding $10 \mathrm{R}$ as mentioned earlier. The overall model wavelength behavior is well-fit with a methane column density of $8 \times 10^{16} \mathrm{~cm}^{-2}$ (column density fit by the model). The regression fit to the weak FUV eclipse signal is not sensitive to the methane column density. However, we have previously modeled the FUV spectrum of 13 December 2004 in Paper 1 and found a methane column density of $3 \times 10^{16} \mathrm{~cm}^{-2}$. Additionally, the $\mathrm{CH}_{4}$ column abundance inferred from the airglow at $1000 \mathrm{~km}$ for T57 in Paper 3 was $2 . \times 10^{16} \mathrm{~cm}^{-2}$, which is the formula that was used in West et al. [2012] for the analysis of the same eclipse emissions. This is about a factor of 2 less than what is reported from occultation in Shemansky et al. [2005], but it should be emphasized that the occultation experiment measures the full slant column abundance through the atmosphere whereas the $\mathrm{CH}_{4}$ column retrieved from the airglow analysis is sensitive to attenuation from the tangent altitude (where the airglow is excited) to the spacecraft.

[28] The failure of the modeled intensity peaks to match the LBH and N I features at short FUV wavelengths (1200$1500 \AA$ ) may indicate proton impact mean energy with larger than $5 \mathrm{keV}$ mean impact energy, producing a different 

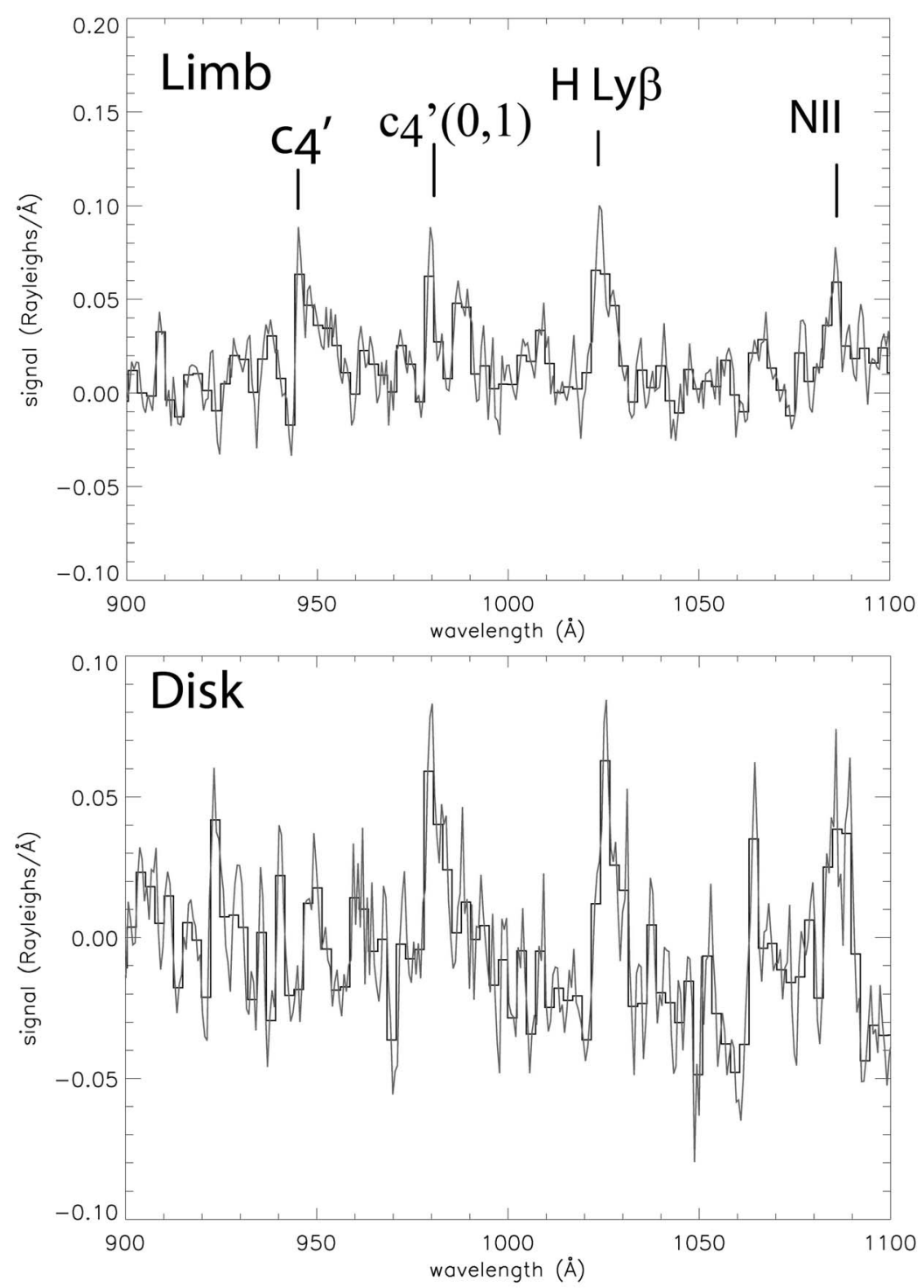

Figure 7. The EUV spectra of the limb and disk of Titan during the solar eclipse of Titan by Saturn. The strong emission features of $c_{4}^{\prime}{ }^{1} \sum_{u}^{+}\left(\mathrm{v}^{\prime}=0\right) \rightarrow \mathrm{X}\left(\mathrm{v}^{\prime \prime}=1\right)$ at $980 \AA, \mathrm{H}$ Ly $\beta$ at $1025 \AA$, N II $(1085 \AA)$ are indicated in both limb and disk. The limb spectrum also shows the blended $c_{4}^{\prime 1} \sum_{u}^{+}\left(\mathrm{v}^{\prime}=4,3\right) \rightarrow \mathrm{X}\left(\mathrm{v}^{\prime \prime}=3,2\right)$ molecular bands near $945 \AA$. The EUV spectra are smoothed by 3-pixels in red, and the nsum data $=4$ (average sum of 4 pixels per point) is shown in black in the histogram mode.

relative set of intensities than measured in our laboratory. It is also possible that having different impacting species (such as $\mathrm{H}_{2}^{+}, \mathrm{He}^{+}$and water group ions- $\mathrm{O}^{+}, \mathrm{OH}^{+}$) will influence these ratios [Sittler et al., 2010a]. As for electrons, the LBH relative band intensities are expected to closely follow Franck-Condon factors at all energies except for collisional cascade from the $\mathrm{a}^{\prime}$ and w-states [Ajello et al., 2010, 2011a]. The total eclipse intensity for the combined limb and disk data is $130 \mathrm{R}$ of which $115 \mathrm{R}$ arises from disk + limb H Ly $\alpha$. The composite intensity for the entire wavelength range found at this modeling stage is $172 \mathrm{R}$, which is $25 \%$ too large to match the data. We find the eclipse data is not of sufficient spatial resolution to distinguish limb and disk or to guarantee a filled FOV for the pixels chosen as we can assure from other nightside observations at much closer distance as described in section 4.0 .
[29] Let us consider the data on strong atomic lines from EUV and FUV dark disk and limb spectra individually. There are strong spectral features of $\mathrm{H} \mathrm{Ly} \alpha(1216 \AA)$ and $\mathrm{H} \mathrm{Ly} \beta$ $(1025 \AA)$ in the FUV and EUV, principally from interplanetary $\mathrm{H}$ solar fluorescence on the limb and from Titan reflection of interplanetary hydrogen on the dark disk. The FUV H Ly $\alpha$ intensity is $122 \mathrm{R}$ on the dark limb and $32 \mathrm{R}$ on the dark disk, after subtracting the contribution from N I $1200 \AA$ from the wings of $\mathrm{H} \operatorname{Ly} \alpha$. These numbers can be directly compared to calculation from a model presented in West et al. [2012]. West et al. find an intensity of $23 \mathrm{R}$ from reflection of interplanetary $\mathrm{H} \mathrm{Ly} \alpha$ by the dark disk of Titan with an uncertainty of a factor of two, indicating an intensity range of $12 \mathrm{R}$ to $46 \mathrm{R}$. The remaining emission of $9 \pm{ }^{11} \mathrm{R}$ from dark disk may be produced by charge exchange with impacting protons, a strong excitation process of Ly $\alpha$ observed in the 
a)

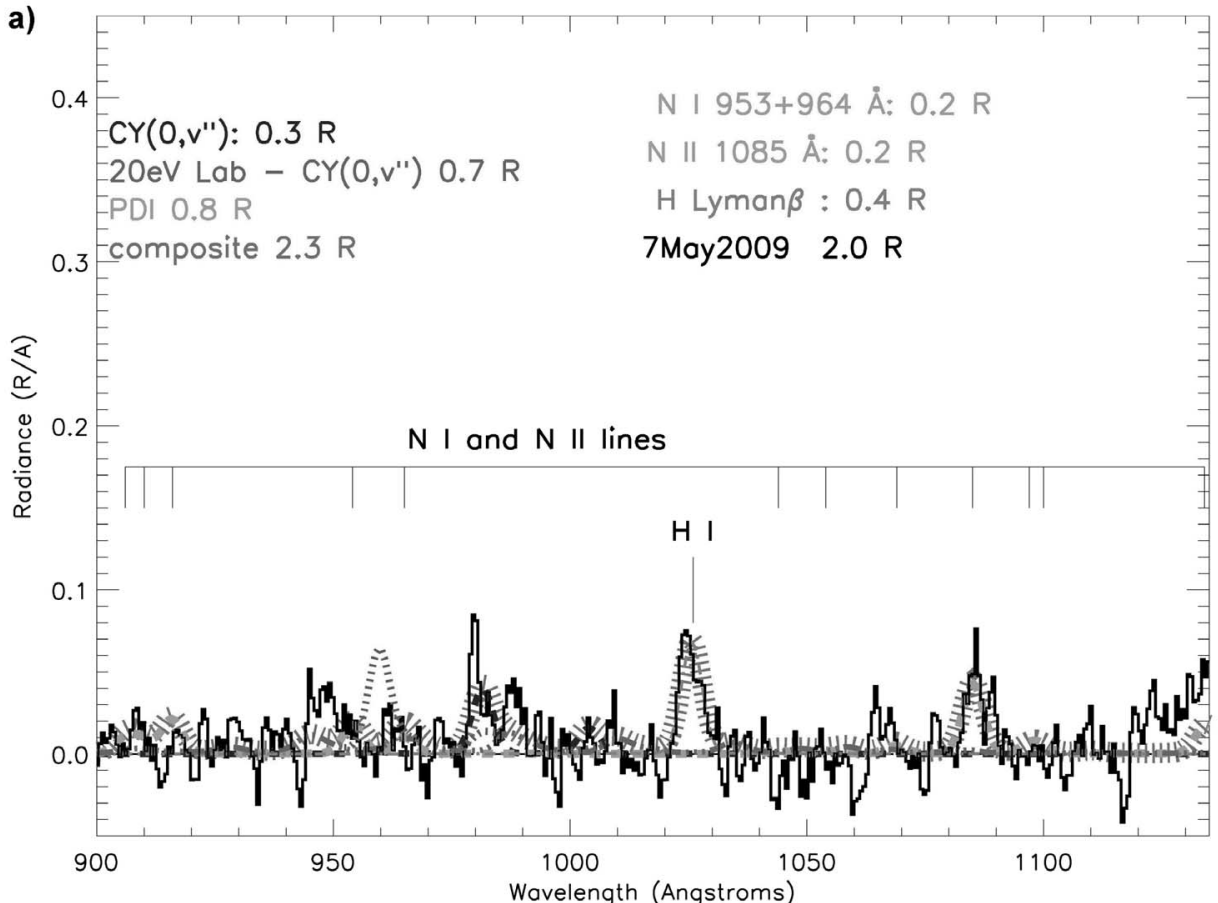

b)
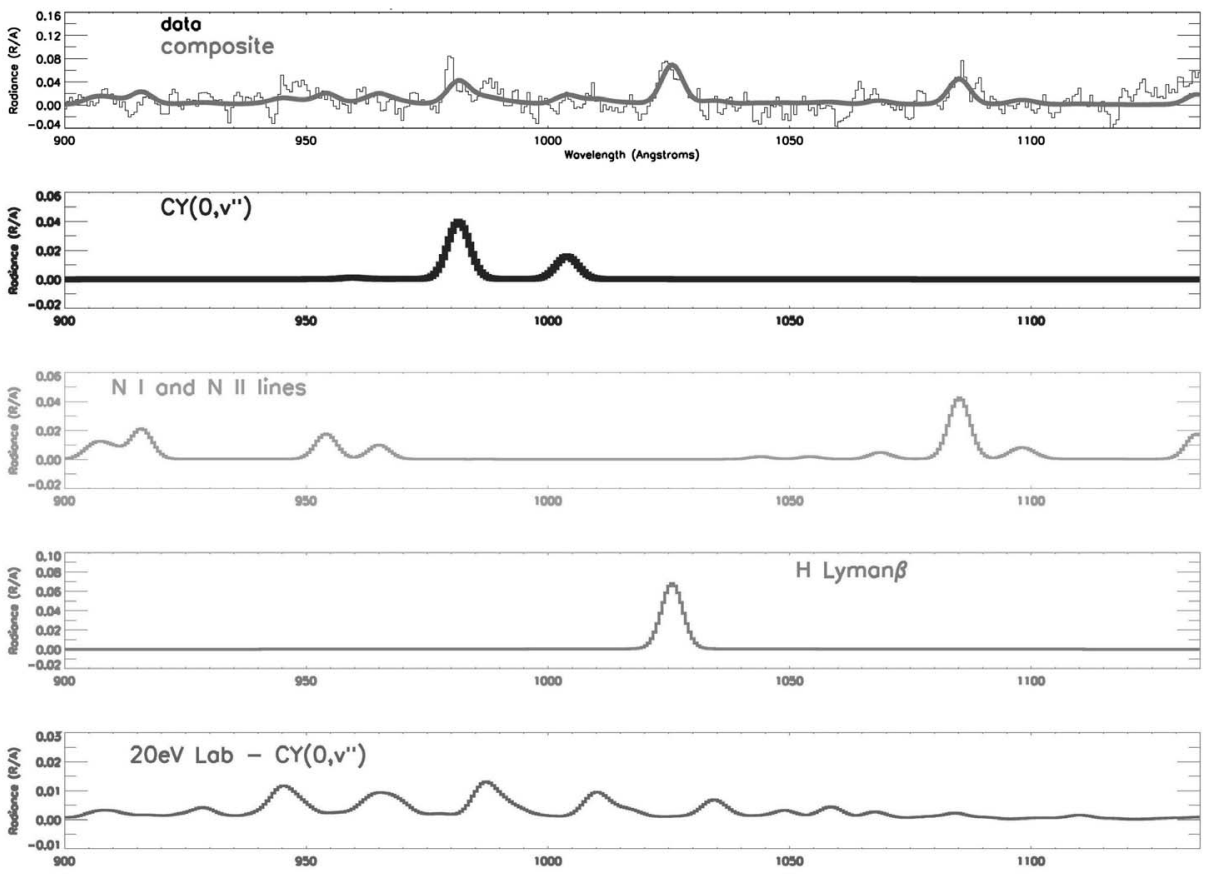

Figure 8. (a) Regression model fit to UVIS EUV eclipse spectrum (black with smooth of 3-pixels) from 7 May 2009. The composite model (orange) includes four independent vectors (totals indicated; see text). We designate: 1) green for the CY $\left.\left(0, \mathrm{v}^{\prime \prime}=0,1,2\right)\right]$; 2) light blue for the NI and NII lines; 3$)$ dark blue for the $\mathrm{H} \mathrm{Ly} \beta$ feature near $1026 \AA$; and 4) red for the $\mathrm{N}_{2} \mathrm{RV}$ bands based on a $20 \mathrm{eV}$ laboratory spectrum. The optically thin $c_{4}^{\prime}(0) \rightarrow \mathrm{X}(0)$ band that was removed from vector 1 (20 eV spectrum) is shown in dottedlight green, and the loss in area of the $c_{4}^{\prime}(0,0)$ band represents multiple-scattering predissociation of $\mathrm{N}_{2}$. (b) The composite model fit to the data, followed by the four individual model fits to the UVIS data for each vector. The individual panels are: 1) data in black and composite model fit orange; 2) the CY bands for $\mathrm{v}^{\prime}=0, \mathrm{v}^{\prime \prime}=0.1 ; 3$ ) the NI,II lines in same PDI ratios as in Stevens et al. [2011]; 4) Lyman- $\beta$; and 5) the $\mathrm{RV}$ bands of $\mathrm{N}_{2}$ at $20 \mathrm{eV}$ from electron impact measured in the laboratory. The strongest are $\mathrm{b}^{1} \Pi_{u}, \mathrm{~b}^{\prime}$ ${ }^{1} \sum_{u}^{+}, c_{4}^{\prime 1} \sum_{u}^{+} \rightarrow \mathrm{X}^{1} \sum_{g}^{+}$ 


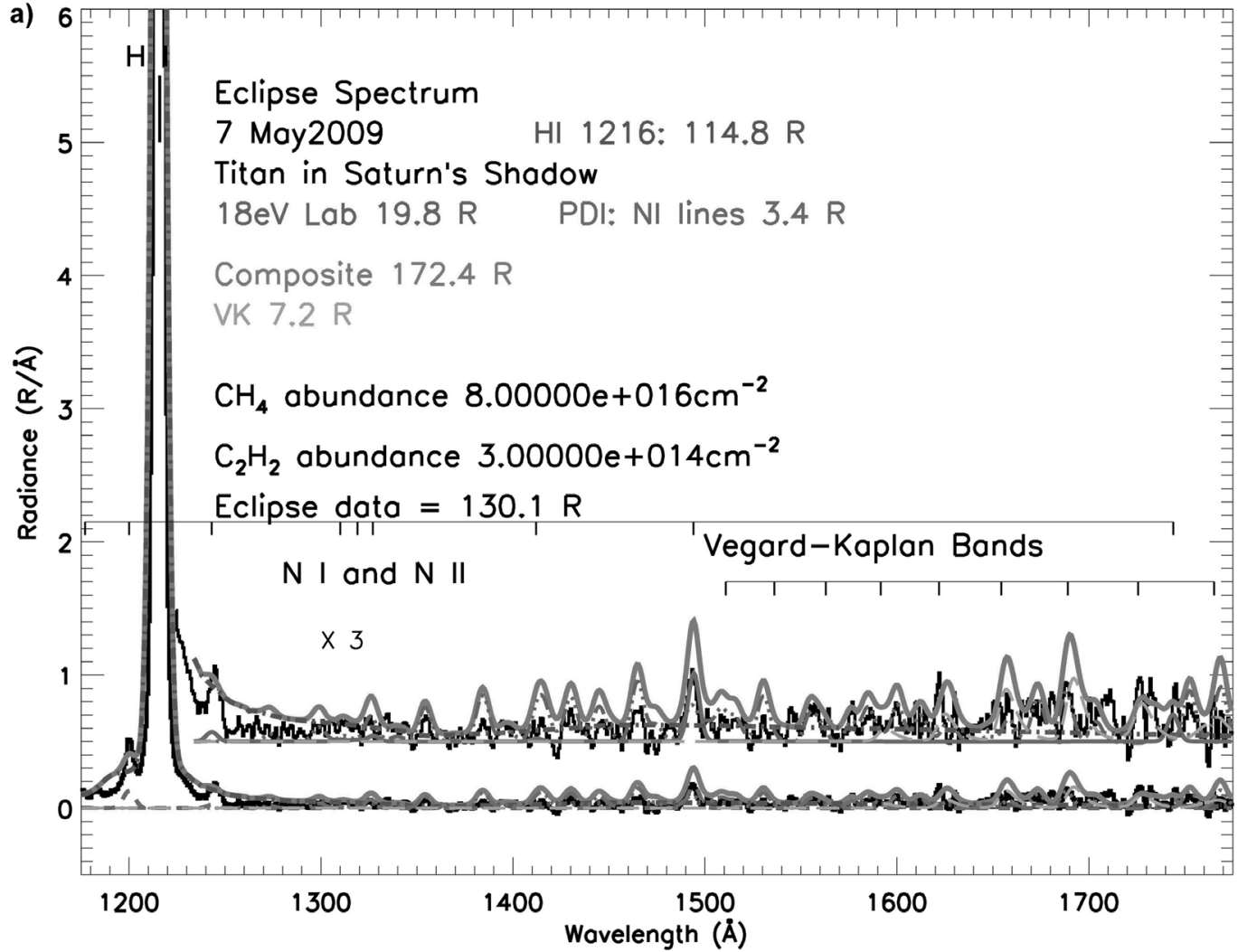

b)
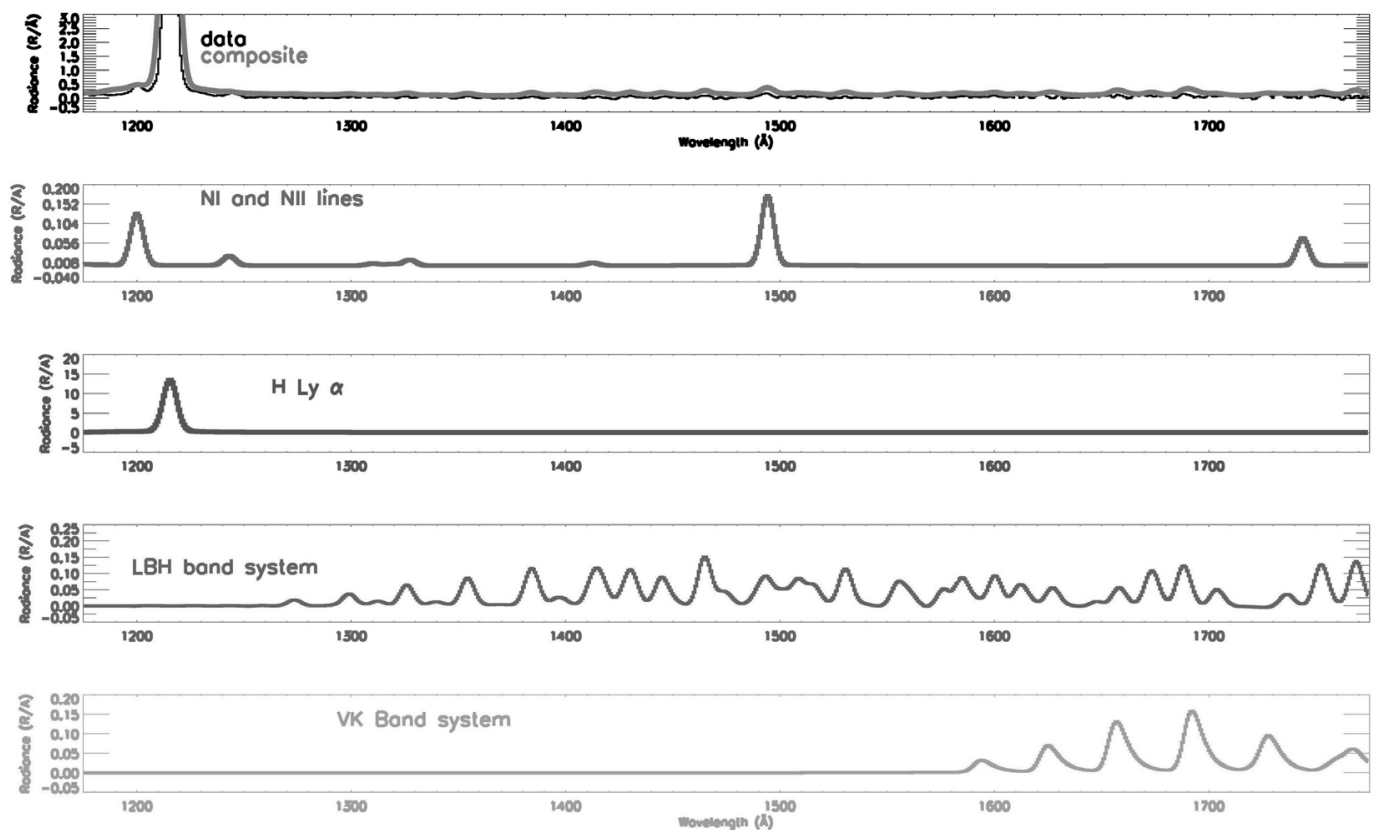

Figure 9. (a) Regression model fit (orange) to the FUV eclipse spectrum (black) from 7 May 2009 in black (smooth of 3-pixels). The composite model (orange) includes four independent vectors (totals indicated; see text). We designate: 1) red for the LBH spectrum); 2) purple for the NI and NII lines; 3 ) green for the H Ly- $\alpha$ feature near $1216 \AA$; and 4) light blue for the $\mathrm{N}_{2}$ VK bands based on a model spectrum [Stevens et al., 2011]. (b) The data composite regression (orange) fit to the data (black) in the first panel followed by four panels of individual modeling vectors. The regression model (orange) in the first panel includes: 1) N I spectrum from dissociative ionization (same ratios as PDI) of $\mathrm{N}_{2}$ in purple in the second panel; 2) the $\mathrm{H} \mathrm{Ly} \alpha$ feature at $1216 \AA$ in green in the third panel; 4) the $\mathrm{LBH} 20 \mathrm{eV}$ laboratory spectrum in red in the fourth panel; and 5) the VK band system in light blue in the fifth panel. 
laboratory [Ajello et al., 2011b] and possibly a small amount also from interplanetary H Ly $\alpha$ along the line of sight from the spacecraft to Titan. A discussion of the wide range of uncertainty in the contribution to the $\mathrm{H} \operatorname{Ly} \alpha$ signal from charge exchange is given in West et al. [2012].

[30] The ratios of N I $1200 \AA / \mathrm{N}$ I $1493 \AA$ (including some contribution from the LBH bands at $1493 \AA$ ) may be indicative of characteristic of either electron impact induced fluorescence or proton induced fluorescence of $\mathrm{N}_{2}$. These laboratory-based optically thin ratios are similar and have been found to be 2.4 for electron impact excitation at $100 \mathrm{eV}$ [Ajello and Shemansky, 1985] and 2.0 for proton induced fluorescence at $5 \mathrm{keV}$ [Ajello et al., 2011b]. The energy dependence of atomic line ratios from electron dissociative processes are typically found to be constant to about $20 \%$ [Ajello and Shemansky, 1985; Ajello and Franklin, 1985]. The measured emission line multiplet ratios are $\sim 3$ on the $\operatorname{limb}(3.10 \mathrm{R} / 1.03 \mathrm{R})$ and disk (1.99 R/0.64 R). The published N I $1200 \AA / \mathrm{N} \mathrm{I} 1493 \AA$ limb and disk observations with a ratio near 2 (Papers 1 and 3 ) were affected by $\mathrm{CH}_{4}$ absorption. We consider this ratio again in another dark side observation 30 times closer to Titan in section 4 .

\section{Observations of the Lower Atmosphere Below 1000 km MRH}

[31] We show in Figure 10 (bottom) the geometry for the Titan observation in F3 at time of record 0 and record 1 with the EUV slit nearly perpendicular to the limb. The start time of record 0 occurs at 20 April 2099 at $1^{\mathrm{h}} 09^{\prime} 8^{\prime \prime}$ and the start time of record 1 was exactly $4^{\prime}$ later at $1^{\mathrm{h}} 13^{\prime} 8^{\prime \prime}$. The slit image is continuously moving as the UVIS slit pans higher into the upper atmosphere with time. The EUV spectrum of F3 for the dark limb is shown for record 1 (top) and the FUV spectrum for record 0 (middle) on 2009-DOY110 of Figure 10. The F3 observation took place at a closer range distance of the spacecraft to Titan than the other 70 Cassini spacecraft observations. F3 acquired four records of Titan at spacecraft distances of 15,557 km, $16827 \mathrm{~km}, 18,106 \mathrm{~km}$ and $19,392 \mathrm{~km}$ representing a factor of $\sim 30-40$ closer than the eclipse, ensuring a filled FOV for each pixel of either the disk or limb. The F3 EUV observation was composed of four 4-min records. We selected record 0 and record 1 for discussion in the FUV and EUV, respectively, since the UVIS slit shown in Figure 10 (bottom) remains partially on the Titan disk and lower atmosphere for the first two records only. We follow upon the discussion with a regression analysis of record 1 for both the EUV and FUV. The orientation of the slit is such that pixel 0 is at the highest altitude and pixel 59 is on the surface (below disk). The relative spacecraft motion moves both the UVIS slits swiftly upwards through the upper atmosphere of Titan from 556 to $818 \mathrm{~km}$ for pixel 0 of record 0 and from $818 \mathrm{~km}$ to $1111 \mathrm{~km}$ for the four minutes of record 1 . Numerically larger pixel numbers are at lower attitudes with pixel 33 on the surface-tangent at the start of record 0 and with pixel 46 on the surface-tangent at the start of record 1 . We choose to use only a subset of the 60 spatial pixels of the slit for record 1 that remain on the dark disk or dark limb for the entire 4 min, pixels 57 to 59 for the disk ( $\mathrm{S} / \mathrm{N}$ is insufficient for dark disk regression analysis but indicates same spectral features as the limb) and pixels 0 to
44 for the dark limb. The EUV signal for dark limb observations are strong on all four records throughout the thermosphere region with MRH from 300 to $1500 \mathrm{~km}$. The limb SZA's for F3 record 0 and 1 are about $123^{\circ}$, indicating that the topside ionosphere $(\sim 1200 \mathrm{~km})$ is in darkness from solar XUV. We very carefully calculated the shadow heights for every pixel element on record 0 and 1 . We find, for the F3 observation, that the sun's light will not illuminate any portion of Titan's atmosphere below $4000 \mathrm{~km}$ along the Cassini line of sight for either the EUV field of view (FOV) with an opaque altitude of $800 \mathrm{~km}$ or the far FUV FOV with an opaque altitude of $350 \mathrm{~km}$, since the shadow height altitude remains above $4000 \mathrm{~km}$ for all 60 pixels at their respective MRH values. The region from $800-1500 \mathrm{~km}$, representing the topside ionosphere, is in darkness, and the observed EUV spectra mainly derive excitation from plasma impact.

[32] We will further analyze record 1 limb spectrum. The emission MRH altitudes for the limb spectra of record 1 observed by the EUV UVIS spans most of the entire ionosphere from grazing the surface at $0 \mathrm{~km}$ for pixel 42 at the start of record 1 to $797 \mathrm{~km}$ for pixel 0 and completing record 1 at $1089 \mathrm{~km}$ for pixel 0 at the end of $4 \mathrm{~min}$. We have constructed for the F3 an average dark limb spectrum spanning the ionosphere. The Titan atmosphere will transmit to Cassini UVIS EUV limb spectra from the MRH altitude for pixel line-of-sight (LOS) distances above $800 \mathrm{~km}$. Thus the spectra arising from MRH altitudes below $800 \mathrm{~km}$ will contain lineof-sight (LOS) spectra from volume elements of spherical shells of the atmosphere above $800 \mathrm{~km}$ altitude. The EUV darkside limb spectrum of record 1 (shown in Figure 10, top) is smoothed by seven pixel elements and shows about 8 identifiable features with peak emission rates at the limb of $\sim 0.1 \mathrm{R} / \AA$ at about of $25 \%$ of the peak EUV intensity emission rate of $\sim 0.4 \mathrm{R} / \AA$ for the same spectral features observed for the dayglow limb observation of 22 June 2009, F14, at $800-1000 \mathrm{~km}$ and about twice the intensity of the eclipse EUV observation shown in Figure 8 . Figure 10 shows the following emission features: $\mathrm{N} \mathrm{II}\left({ }^{3} \mathrm{P} \leftarrow{ }^{3} \mathrm{P}^{\mathrm{o}}\right)$ at $916 \AA, \mathrm{c}^{\prime}{ }_{4}(3,2$; $4,3)$ at $943 \AA, N I\left({ }^{4} \mathrm{~S}^{\mathrm{o}} \leftarrow^{4} \mathrm{P}\right)$ at $954 \AA, c_{4}^{\prime}(0,1)$ at $980 \AA, c_{4}^{\prime}(0,2)$ at $1003 \AA, \mathrm{H}$ Ly $\beta$ at $1025 \AA, \mathrm{N} \mathrm{II}\left({ }^{3} \mathrm{P}^{2}{ }^{3} \mathrm{D}^{\circ}\right)$ at $1086 \AA$ and $\mathrm{N}$ $\mathrm{I}\left({ }^{4} \mathrm{~S}^{\mathrm{O}} \leftarrow{ }^{4} \mathrm{P}\right)$ at $1134 \AA$.

[33] Similarly, we show in Figure 10 (middle) the F3 FUV spectrum of record 0 (smoothed by 7 pixels for improved $\mathrm{S} / \mathrm{N}$ ) for the dark limb over-plotted with a laboratory protonexcited LBH spectrum and a model VK spectrum. Once again we use only a subset of the 60 spatial pixels of the FUV slit for record 0 choosing pixels that remain on the dark limb for the entire $4 \mathrm{~min}$, pixels $0-32$ for the limb. The vertical resolution of the FUV and EUV spatial pixels are less than $50 \mathrm{~km}$. The FUV limb spectrum clearly shows both LBH and VK band system vibrational features with a spectral intensity about of $20 \%$ of the intense dayglow limb observation of F14. The brighter LBH features have a peak intensity of $\sim 0.3 \mathrm{R} / \AA$ versus $\sim 1.5 \mathrm{R} / \AA$ on $\mathrm{F} 14$ (and versus $0.1 \mathrm{R} / \AA$ during the eclipse). The $\mathrm{H}$ Ly $\alpha$ FUV signal from limb and disk are nearly identical with intensities of $40 \mathrm{R}$ on the limb and $36 \mathrm{R}$ on the disk, indicating that on F3 the $\mathrm{H}$ Ly $\alpha$ comes mainly from Titan atmosphere scattering of interplanetary Ly $\alpha$ [West et al., 2012]. The H Ly $\alpha$ disk and limb intensities of F3 are nearly the same, and similar to the disk observation of the eclipse. 

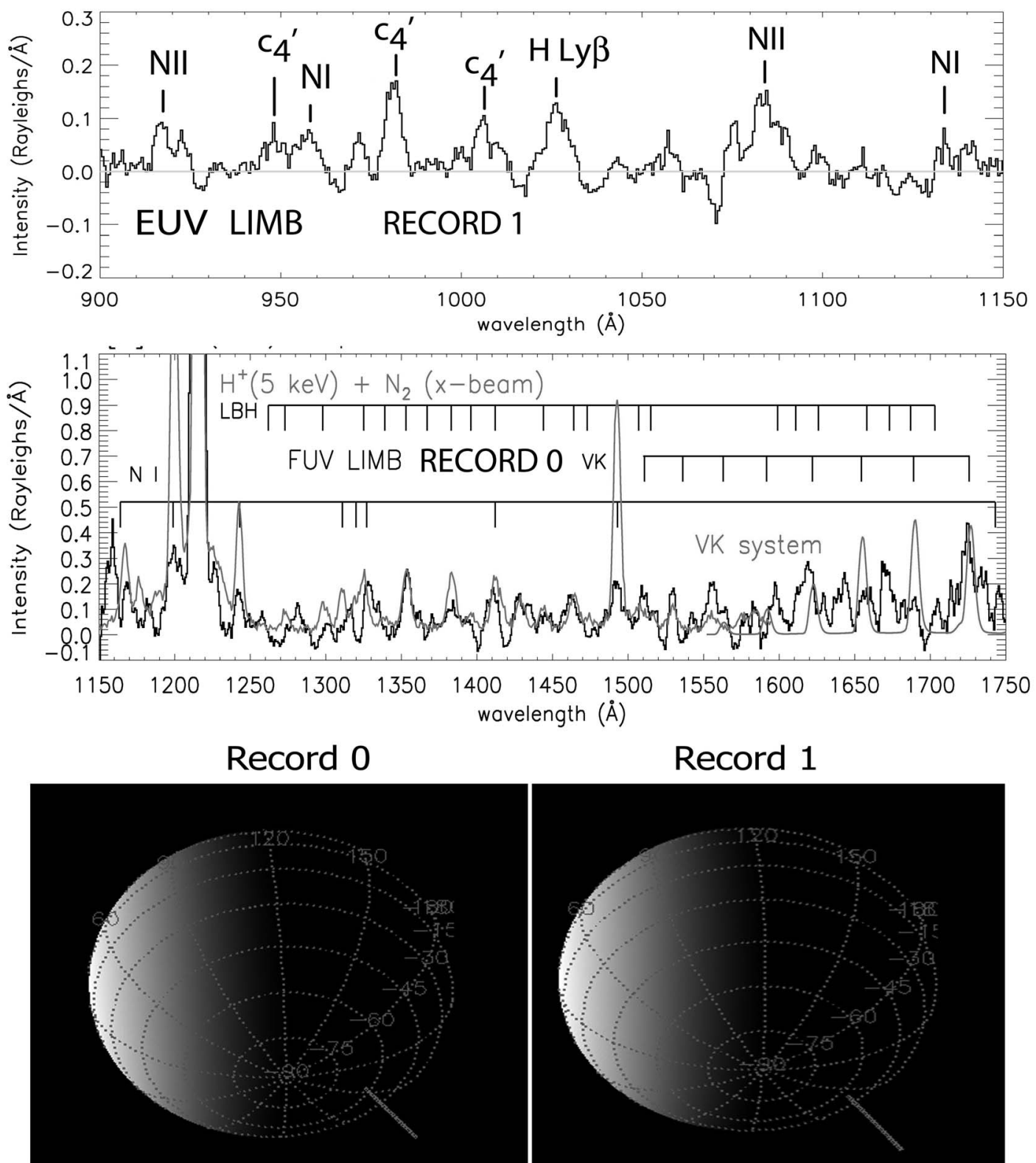

\section{EUV2009_110_01_12 34_UVIS_109TI_FIRLMBAER002_CIRS}

Figure 10. (top) The EUV spectrum of F3 from Record 1 (smooth $=7$ ) is shown for dark limb (pixels 0-45), which is from data cube EUV 2009_110_01_15_34_UVIS_109TI_FIRLMBAER002 CIRS 750_800.sav. The strong features are identified by tick mark positions of NII $(917 \AA), \mathrm{N}_{2} c_{4}^{\prime 1} \sum_{u}^{\mp}$ $\left(\mathrm{v}^{\prime}=\overline{4}, 3\right) \rightarrow \mathrm{X}\left(\mathrm{v}^{\prime \prime}=3,2\right)$ at $945 \AA, \mathrm{N}$ I $(953 \AA), c_{4}^{\prime 1} \sum_{u}^{+}\left(\mathrm{v}^{\prime}=0\right) \rightarrow \mathrm{X}\left(\mathrm{v}^{\prime \prime}=1\right)$ at $980 \AA, c_{4}^{\prime} \sum_{u}^{+}\left(\mathrm{v}^{\prime}=0\right)$ $\rightarrow \mathrm{X}\left(\mathrm{v}^{\prime \prime}=2\right)$ at $1003 \AA \mathrm{H} \mathrm{Ly} \beta$ at $1025 \AA$, N II $(1085 \AA)$, and N I $1134 \AA$. (middle) The FUV spectrum (black) of observation F3 for the dark limb for record 0, which is FUV2009_109_23_06_00_UVIS_109-

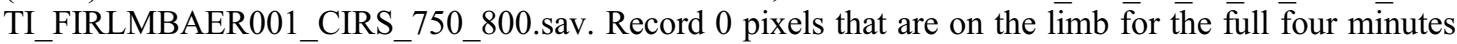
are $0-32$. The overplot in green is a laboratory spectrum of $5 \mathrm{keV}$ proton excited $\mathrm{N}_{2}$ showing the LBH bands, H Ly $\alpha$ and the N I multiplets. The overplot in orange shows the electron excited synthetic model spectrum of the VK bands of $\mathrm{N}_{2}$. (bottom) The geometry of the UVIS observation of F3 for the initial time of Record 0 and Record 1 that starts on 2009 DOY $11001^{\mathrm{h}} 09^{\prime} 08^{\prime \prime}$ for Record 0 at an initial distance of $14925 \mathrm{~km}$ and at 2009 DOY $11001^{\mathrm{h}} 13^{\prime}-08^{\prime \prime}$ for Record 1 at an initial distance of $16,190 \mathrm{~km}$ for the 4-min data records. The location of the slit is near the south pole at about $58^{\circ} \mathrm{S}$. 
[34] There is additional evidence that the FUV Record 0 signals for LBH band features and atomic multiplet wavelengths below $1400 \AA$ arise from a higher and different altitude than the $1493 \AA$ multiplet. The ratio of the NI $1200 \AA / \mathrm{NI}$ $1493 \AA$ integrated intensities are close to 1.5 as the NI $1200 \AA$ mutiplet is weakened at deep layers below $800 \mathrm{~km}$ by $\mathrm{CH}_{4}$ absorption.

[35] We have over-plotted the Titan FUV UVIS spectrum in Figure 10 (middle) with our recent optically thin laboratory FUV proton-induced fluorescence spectrum in green of $\mathrm{p}(5 \mathrm{keV})+\mathrm{N}_{2}$ illustrating strong LBH bands [Ajello et al., 2011b]. Proton impact on $\mathrm{N}_{2}$ molecules to depths of 500$600 \mathrm{~km}$ has been modeled by Cravens et al. [2008, 2009]. Our $5 \mathrm{keV}$ proton impact laboratory spectrum reasonably matches the Titan FUV spectrum for most peaks from 1100 to $1600 \AA$ and in the laboratory the $\mathrm{H} \mathrm{Ly} \alpha$ arises mainly from a charge transfer reaction (or electron capture reaction) with $\mathrm{N}_{2}$. We also show the synthetic spectrum of electron induced fluorescence of the VK bands referenced in Paper 3; this marginal match is well-improved in the regression analysis to follow with a smooth of the data by 3-pixels.

[36] As for the eclipse observation, we now proceed to a regression analysis of the $\mathrm{F} 3 \mathrm{limb}$ observation for record 1 with a smooth of the data by three. We show a limb spectrum for record 1 in Figure 11 for the EUV and in Figure 12 for the FUV. Both data sets are well-matched by a composite regression model. The EUV spectrum is shown in Figure 11 from 900 to $1150 \AA$ with an integrated EUV intensity of $5.5 \mathrm{R}$ compares to a composite fit of $5.0 \mathrm{R}$. These intensities can also be compared to a similar dayglow spectrum of 13 December 2004 analyzed by a regression analysis from 900 to $1140 \AA$ with a measured intensity of $16.6 \mathrm{R}$ and a modeled intensity of $17.5 \mathrm{R}$. For example the $\mathrm{CY}(0,1)$ band has a modeled F3 intensity of $0.9 \mathrm{R}$. compared to $1.5 \mathrm{R}$ in the 13 December 2004 and 22 June 2009 dayglow (and about 4-times the intensity of the eclipse observation). We see approximately a factor of $\sim 2$ 5 differences in the EUV between dayglow and F3 nightglow atomic and molecular intensities. For example, the N II $1085 \AA$ intensity in F3 was $0.7 \mathrm{R}$ compared to $3.9 \mathrm{R}$ on 22 June 2009 and $2.3 \mathrm{R}$ on 13 December 2004. This nightglow spectrum appears to be at least a factor of two brighter than the eclipse spectrum, but the weakness of the eclipse spectrum could be partially due to the filling factor. The importance of the filling factor in the eclipse observations is thoroughly discussed in West et al. [2012].

[37] We show in Figure 14 the regression model of the FUV F3 spectrum from $1175 \AA$ to $1775 \AA$. The main difference between F3 FUV regression model and the eclipse FUV regression model lies in fact that a weak solar scattering vector is useful in the $\mathrm{F} 3$ regression model and may arise from multiple scattering around the moon from the bright disk hemisphere as the Cassini LOS within the thermosphere and exobase region is in darkness. The chi squared regression factor is slightly improved including this weak vector addition in the regression model. It is difficult to choose between the two models (with and without solar scattering) that only differ in the amount of VK emission intensity. The model for the LBH bands predicts the LBH emission rate at $20.1 \mathrm{R}$ and for the VK bands the model projects an emission rate of $2.6 \mathrm{R}$ (9.6 R without long wavelength solar scattering). Stevens et al. [2011] find a peak LBH dayglow intensity of $136 \mathrm{R}$ and a VK dayglow intensity of $67.8 \mathrm{R}$ for F14.
We find an LBH intensity of $43 \mathrm{R}$ in Paper 1 for the 13 December 2004 observation. Taken together, these dayglow changes indicate significant variations in the FUV dayglow for all atomic and molecular emissions, as Figure 5 already demonstrates for the 71 observations considered.

[38] The atomic lines in the FUV dayglow as in the EUV dayglow are about a factor of five stronger than the F3 nightglow intensities. The F3 intensity of atomic N I $1200 \AA$ is $2.9 \mathrm{R}$ compared to an intensity of $1.5 \mathrm{R}$ for N I $1493 \AA$. The ratio of a factor of two for the intensities of N I $1200 \AA / \mathrm{N} \mathrm{I}$ $1493 \AA$ is the same ratio as found by Stevens et al. [2011] in the dayglow from the fluorescent emission attributed to electron impact reduced by the effect of $\mathrm{CH}_{4}$ absorption. The $\mathrm{F} 3$ ratio is more characteristic of proton impact as measured in the laboratory by Ajello et al. [2011b]. The overall regression model wavelength behavior is well fit with a methane column density of $2 \times 10^{16} \mathrm{~cm}^{-2}$. The absorption cross section of methane is nominally $2 \times 10^{-17} \mathrm{~cm}^{2}$ in the FUV at $1200 \AA$, indicating only a small $10 \%$ absorption slant transmission effect to match the F3 EUV observation that occurs at about $900 \mathrm{~km}$ altitude on Titan, a representative mean altitude for the F3 EUV and FUV spectra [Cravens et al., 2009]. The total F3 integrated intensity for the data is $113.2 \mathrm{R}$, which can be broken down using the model: 1) $59 \mathrm{R}$ arises from $\mathrm{H}$ Ly $\alpha$ (including the broad wings shown in Figure 14, top) and 2) $21.5 \mathrm{R}$ from long FUV wavelength multiple solar scattering to the dark side. The composite intensity for the entire wavelength range found at this modeling stage is $124 \mathrm{R}$, which well-matched the composite data intensity.

[39] The main difference between F0 (another close encounter darkside observation from a spacecraft to Titan distance of 19,970-23,866 km) is a weaker airglow FUV spectrum of limb than F3 (and F14) $(\sim 1 / 2(\sim 1 / 10)$ of the intensity of EUV and FUV airglow spectrum of F3 (F14)). We note also that the F0 disk observation positions the UVIS slit near the equator $\left(8^{\circ}-20^{\circ} \mathrm{S}\right)$ and $\mathrm{F} 3$ is more polar $45^{\circ}-60^{\circ} \mathrm{S}$. The EUV limb airglow spectrum of F0 is equally intense as F3 is for the limb (compare Figures $3 a$ and 5 with Figure 9).

[40] The latitude location of the F3 EUV and FUV emissions are similar to the near-polar latitude found by Kliore et al. [2008] for the strong lower altitude sporadic layer of peak electron density layer near $500 \mathrm{~km}$ from a radio occultation measurement on orbits T12 and T14. The latter radio occultation occurs with southern polar latitudes nearly the same as the UVIS F3 observation location [Cravens et al., 2009].

[41] The ISS photometric filtered image [West et al., 2011, 2012] indicated circum-Titan plasma particle excitation of a nightglow producing the $300-1100 \mathrm{~nm}$ visible-optical-near IR spectra with maximum intensity on a line from Titan to the sun through the Saturn disk. We have possibly identified the spectral features from our laboratory work on $\mathrm{N}_{2}$ electron impact fluorescence [Mangina et al., 2011]. The laboratory spectrum from 2000 to $11,000 \AA$ is identified as molecular emission bands of the First Positive Band System (1PB) $B$ ${ }^{3} \Pi_{\mathrm{g}}^{+}\left(\mathrm{v}^{\prime}\right) \rightarrow A^{3} \sum_{\mathrm{g}}^{+}\left(\mathrm{v}^{\prime \prime}\right)$ and the Second Positive Band System (2PB) $C^{3} \Pi_{\mathrm{u}}\left(\mathrm{v}^{\prime}\right) \rightarrow B^{3} \Pi_{\mathrm{g}}\left(\mathrm{v}^{\prime \prime}\right)$ of $\mathrm{N}_{2}$, the First Negative Band System (1NB) $B^{2} \sum_{u}^{+}\left(\mathrm{v}^{\prime}\right) \rightarrow X^{2} \sum_{g}^{+}\left(\mathrm{v}^{\prime \prime}\right)$ and Meinel Band System (MB) $A^{2} \Pi_{u}\left(\mathrm{v}^{\prime}\right) \rightarrow X^{2} \sum_{g}^{+}\left(\mathrm{v}^{\prime \prime}\right)$ of $\mathrm{N}_{2}^{+}$ions, as well as line emissions of N (N I) and $\mathrm{N}^{+}(\mathrm{N}$ II) in the Visible-Opticalnear IR. 
a)

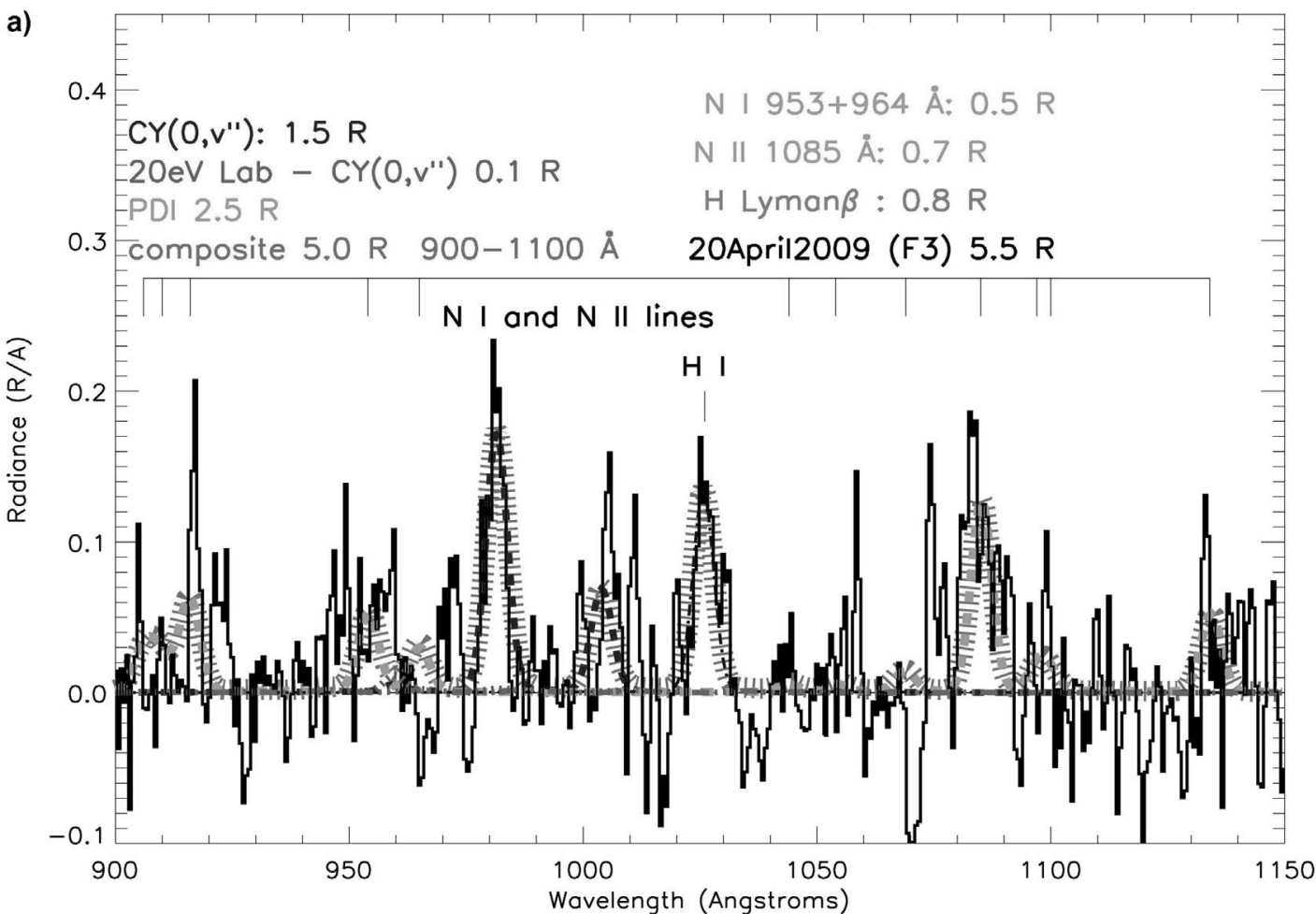

b)
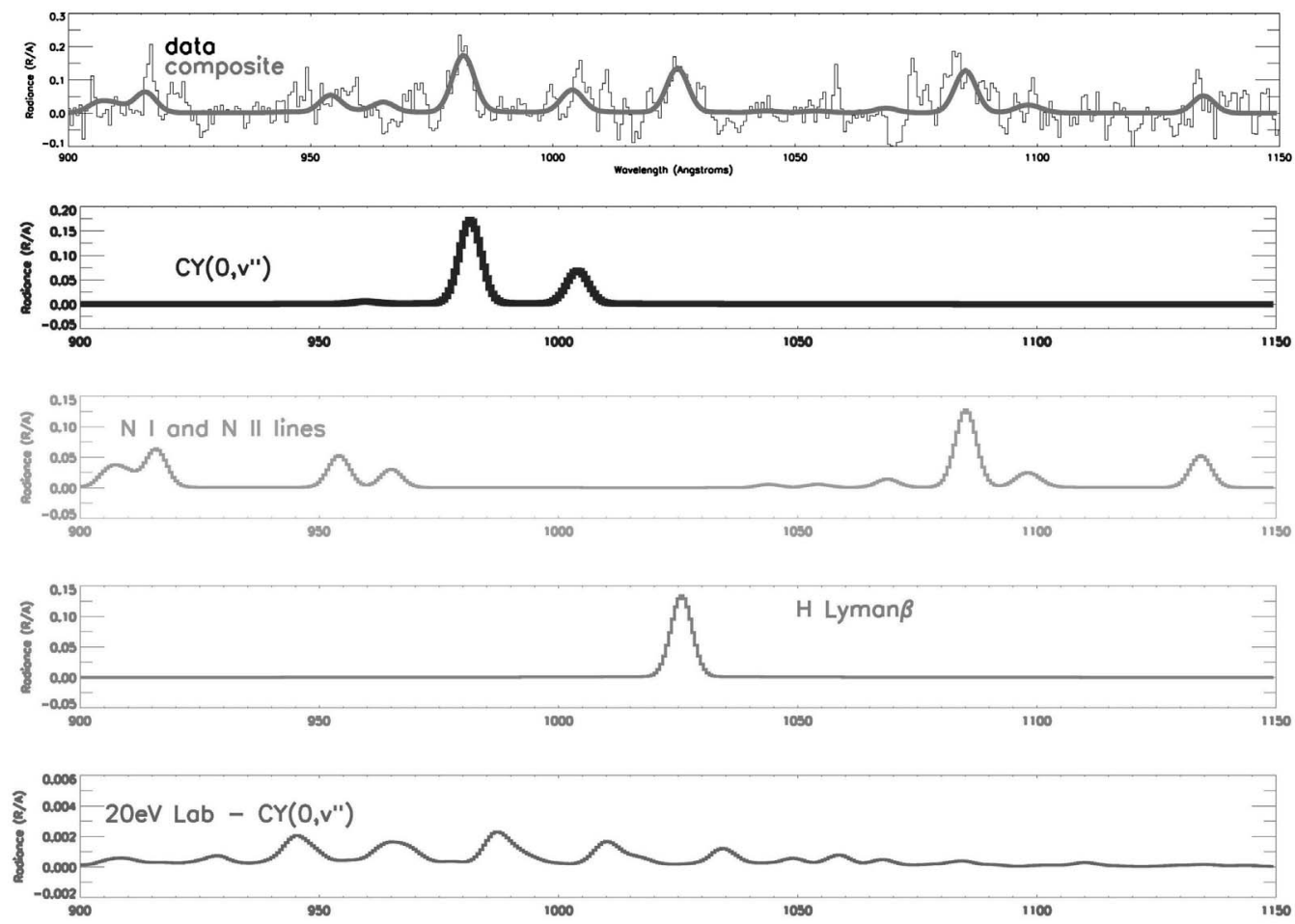

Figure 11. (a) Regression model fit to UVIS EUV F3 Record 1 limb spectrum (black, smooth is 3-pixels) from 20 April 2009. The composite regression model (orange) includes four independent vectors (total integrated intensity indicated for each vector; see text). (b) The composite model fit (orange) to the data (black) followed by the four individual fits to each vector. The individual panels are: 1) data in black and composite model fit in orange in the first panel; 2) the CY bands for $\mathrm{v}^{\prime}=0, \mathrm{v}^{\prime \prime}=0,1,2$ in green in the second panel; 3) the N I,II lines in same PDI ratios as in Stevens et al. [2011] in light blue in the third panel; 4) Lyman- $\beta$ in dark blue in the fourth panel; and 5) the RV bands of $\mathrm{N}_{2}$ at $20 \mathrm{eV}$ from electron impact measured in the laboratory $\mathrm{b}^{1} \Pi_{u}, \mathrm{~b}^{\prime}{ }^{1} \sum, \mathrm{c}^{1} \sum \rightarrow \mathrm{X}^{1} \sum$ in red in the fifth panel. 


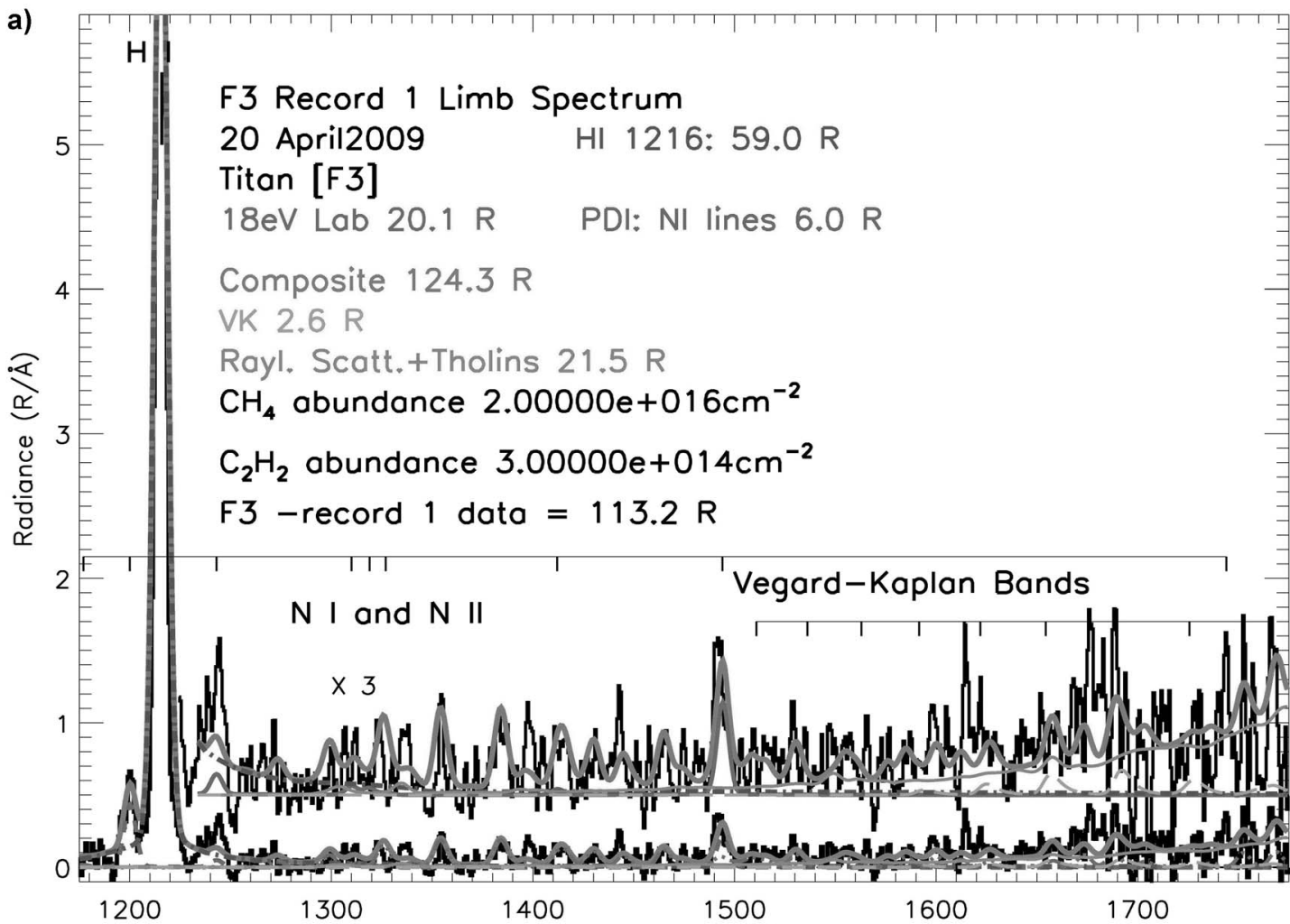

b)
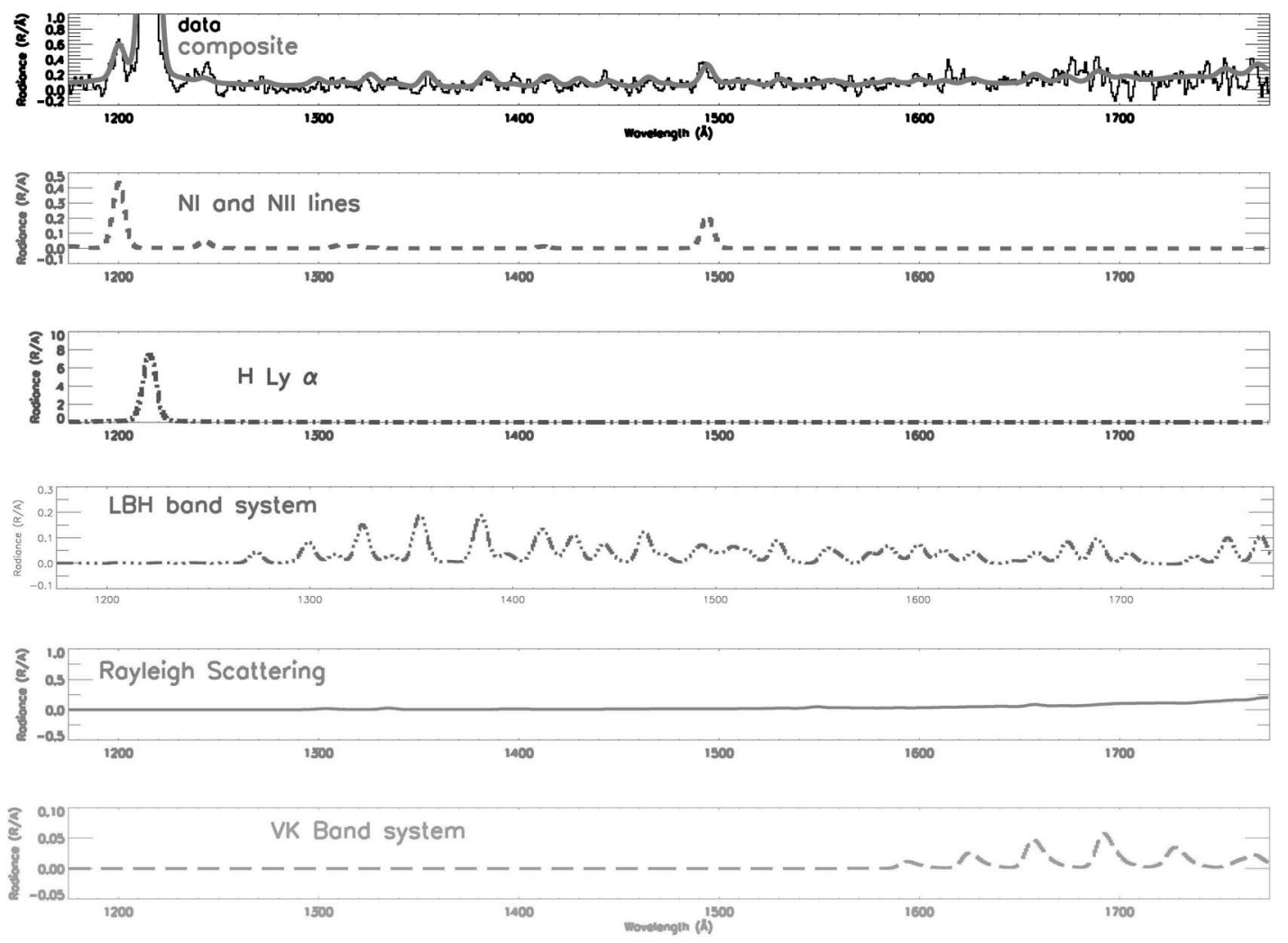

Figure 12 
[42] Energetic protons and oxygen ions have been observed in Saturn's outer magnetosphere and can precipitate into Titan's atmosphere where they deposit energy, ionize, and drive ionosphere chemistry. Ion production rates caused by this precipitation have been calculated using fluxes of incident $27 \mathrm{keV}$ to $4 \mathrm{MeV}$ protons measured by the Cassini MIMI Instrument [Cravens et al., 2008]. These authors find that significant ion production rates exist in the $500 \mathrm{~km}$ to $1000 \mathrm{~km}$ altitude range. This is the MRH altitude range of Record 0 and Record 1 where the UVIS observes significant EUV and FUV night airglow emissions for F0 and F3. The FUV instrument has a sharp transmission decrease below wavelengths of 1400 $\AA$ at $700 \mathrm{~km}$ vertical height for disk viewing and $800 \mathrm{~km} \mathrm{MRH}$ vertical height for slant transmission limb observations. The FUV limb spectrum is optically thin for $\mathrm{CH}_{4}$ absorption for all altitudes (above $350 \mathrm{~km}$ ) and above a wavelength of $1400 \AA$. We surmise that the UV airglow spectra in Figure 11 and Figure 12 arise from all MRH altitudes either above 350 or above $800 \mathrm{~km}$ depending on spectral wavelength (see shadowheight discussion in sections 1 and 5).

\section{Regression Model of 25 March 2008 UVIS Observation}

[43] The 25 March 2008 UVIS EUV observation of Titan from a distance of $260,671 \mathrm{~km}$ and target phase angle of $111.6^{\circ}$ is shown with slit location in Figure 13 for the midtime of record 0 . All the 60 pixels of the FOV of both the UVIS slits simultaneously contain either the bright limb, bright disk, dark disk or the dark limb. We have an opportunity to analyze the average spectrum for each of these spectral pixel sets, separately. For that purpose, we also show in Figure 13 (bottom) the integrated intensity signal for all 60 spatial pixels and 20 records for several key emissions: $\mathrm{H}$ Ly $\alpha$, NI $1494 \AA$ and the particle scattering, the latter over the wavelength range from 1520 to $1910 \AA$. It appears that the radius of the planet is different for each emission, since the limb altitude (pixel) for peak signal depends on wavelength. The smallest altitude ( $\sim-200 \mathrm{~km}$ above the surface) occurs for the haze producing the particle scattering in the long wavelength range of the FUV. The next altitude profile shown is the airglow layer from $\mathrm{N}_{2}$ PDI and secondary electron ionization-excitation near $1000 \mathrm{~km}$, and finally the H Ly $\alpha$ emission profile near the exobase at $1400 \mathrm{~km}$. We also note that though the disk surface is in darkness in Figure 13 (bottom), that the upper atmosphere is catching some sun light at the maximum SZA of $\sim 115^{\circ}$. We see secondary minima in each of the three emission features near the dark side limb and continue to denote this lighting condition as atmospheric twilight. The 25 March 2008 observations provide verification of the importance of different shadow height calculations for the different FUV wavelength ranges.

[44] First, we analyzed the two EUV spectra for the bright limb and the bright disk (not shown). In Figure 14 we show the EUV spectra for the dark limb and dark disk. Each of these spectra were analyzed according to regression models of $\mathrm{N}_{2}$ emission spectra as presented in Papers 1 and 2, using the Jet Propulsion laboratory (JPL) laboratory e $+\mathrm{N}_{2} 18 \mathrm{eV}$ spectrum (with the $\operatorname{CY}\left(0, v^{\prime \prime}=0,1,2\right)$ bands removed), a spectrum too low in impact energy to contain N I, II multiplets. This modeling technique is the same approach as Papers 1 and 2 and a different approach than in Paper 3 where the different features arising from either photoelectron excitation or photo-fragmentation were fit separately. The optically thin $18 \mathrm{eV}$ electron impact laboratory fluorescence spectrum (simulating the airglow spectrum of the $\mathrm{N}_{2}$ bands) was measured at $0.5 \AA$ FWHM with the ad hoc removal of the optically thick $c^{\prime}\left(0, v^{\prime \prime}=0-2\right)$ progression, which dominates the optically thin laboratory spectrum (in orange in dots) in the regression model in Figure 14.

[45] The EUV dark limb and dark spectra are fit between 850 and $1150 \AA$ and all of the features are convolved with the UVIS instrument line shape before inclusion into the leastsquares fitting algorithm. The different spectral components are listed below:

[46] 1) The $C Y\left(0, v^{\prime \prime}\right)$ bands. The $(0,0)$ and $(0,1)$ bands near 958 and $980 \AA$, respectively, are fit together but we use the $(0,1) /(0,0)$ ratio of 40 calculated under limb viewing conditions from a multiple scattering model [Stevens, 2001], thereby rendering negligible the contribution from the optically thick $(0,0)$, as observed [Ajello et al., 2007]. The $(0,2)$ band near $1003 \AA$ is fit separately and the $\left(0, v^{\prime \prime}>2\right)$ bands are weak and are part of the $18 \mathrm{eV}$ laboratory spectrum used as the main fitting spectrum for the regression analysis (Paper 1).

[47] 2) The strong dipole-allowed transitions of $N_{2}$ in the $E U V$. The electronic transitions proceed from the $X^{1} \sum_{g}^{+}$ ground-state to several closely spaced $(\sim 12-15 \mathrm{eV}) \mathrm{RV}$ states, which are the source of the molecular emissions in the EUV observed by spacecraft and have recently been studied in our laboratory at medium-to-high spectral resolution $(\lambda=0.1 \AA$ FWHM). The $18 \mathrm{eV}$ laboratory spectrum in the regression model contain optically thin emissions from these three states to the ground state, except for most transitions terminating in $\mathrm{v}^{\prime \prime}=0$, which are lost to multiple scattering. Other electronic states contribute weaker emission bands found in the EUV spectrum (Ajello et al., submitted manuscript).

[48] 3) Excess of $C Y\left(v^{\prime}=3,4,6\right)$ bands as found in Paper 3. Since the vibrational features are relatively weak and blended, all three of these progressions are fitted together as one spectral component using the relative cross sections of

Figure 12. (a) Regression model fit to the UVIS FUV F3 Record 1 limb spectrum (black, smooth is 3-pixels) from 20 April 2009 in black. The composite model (orange) includes four independent vectors (totals indicated; see text). We designate: 1) red for the LBH spectrum; 2) purple for the N I and N II lines; 3) green for the H Ly $\beta$ feature near 1026 $\AA$; and 4) light blue for the $\mathrm{N}_{2}$ VK bands based on a model spectrum [Stevens et al., 2011]. (b) The data composite regression (orange) fit to the data (black) in the first panel followed by five panels of individual modeling vectors. The regression model (orange) in the first panel includes: 1) N I spectrum from dissociative ionization (same ratios as PDI) of N2 in purple in the second panel; 2) the H Ly $\alpha$ feature at $1216 \AA$ in green in the third panel; 3) the LBH $20 \mathrm{eV}$ laboratory spectrum in red in the fourth panel; 4) the Rayleigh scattered component described in the fifth panel in dark blue; and 5) the VK band system in light blue in the sixth panel. 

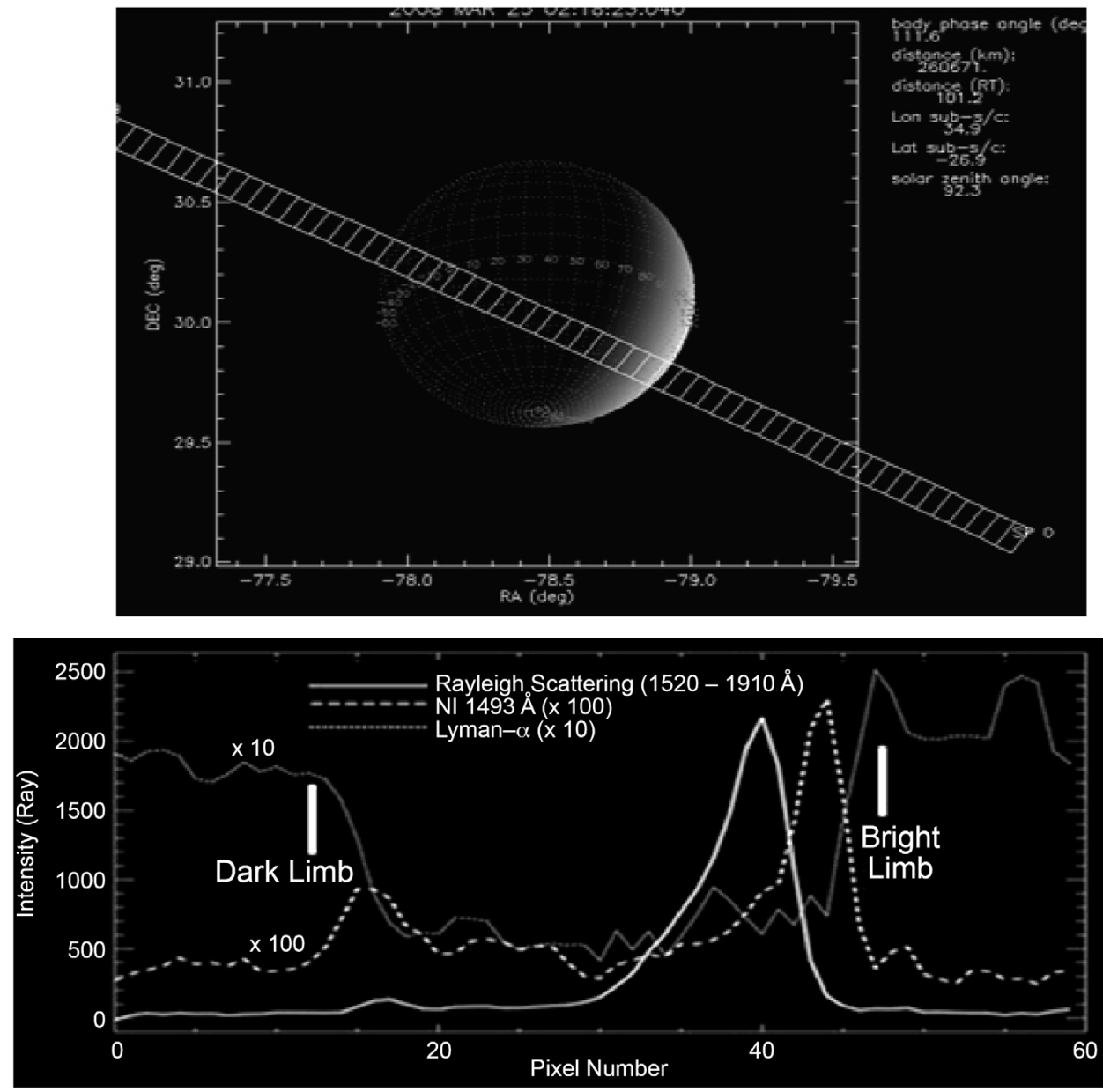

Figure 13. (top) Geometric plot of the Titan 25 March 2008 observation as viewed by the Cassini UVIS EUV slit at mid-time of Record 0 . The UTC mid-time of Record 0 was 25 March $2008,02^{\mathrm{h}} 18^{\prime}$. There were twenty 4.5-min records. (bottom) The integrated intensity signal for each of 60 spatial pixels summed over 20 records for key emissions: H Ly $\alpha$, NI $1494 \AA$ and the particle scattering, the latter over the wavelength range from 1520 to $1910 \AA$.

Ajello et al. [1989, 1998; submitted manuscript, 2012]. To a good approximation the ratio of rotational-vibrational cross sections is independent of electron energy, for electron energies distant from threshold by $\sim 5-20 \mathrm{eV}$. The ratio of relative emission intensities are mainly dependent on the ratio of perturbed emission transition-probabilities for the Rydberg-valence states of $\mathrm{N}_{2}$ [Liu et al., 2005, 2008; Ajello et al., 1989], which are independent of electron energy, since the CY bands are not populated by cascade.

[49] 4) NI and N II multiplets. These features are produced primarily by photo-fragmentation of $\mathrm{N}_{2}$. There are a total of 16 atomic multiplets within the UVIS EUV spectral region. The brightest three atomic features as measured in the laboratory under optically thin conditions are fit individually: N II $1085 \AA$, N I $1134 \AA$ and N II $916 \AA$. The other 13 atomic multiplets in the EUV from $862 \AA$ to $1100 \AA$ are fit as one spectral component according to the laboratorymeasured relative intensities of Samson et al. [1991].

[50] 5) The $H$ Lyman- $\beta$ line at $1026 \AA$. The brightest EUV (850-1150 $\AA$ ) spectrum (not shown) arises from the bright limb with an observed intensity of $22.4 \mathrm{R}$ compared to the bright disk intensity of $10.9 \mathrm{R}$ (half as much as the bright limb). The dark side spectra (850-1150 $\AA$ ) are also about half the brightness with a measured limb brightness of $11.0 \mathrm{R}$ and a measured dark disk brightness of $6.2 \mathrm{R}$ indicated in Figure 14. We believe the dark side spectra for 25 March 2008 arise from a fully illuminated ionosphere at $1000 \mathrm{~km}(800-1200 \mathrm{~km}$ layer profile) altitude. To illustrate this point we show in Figure 15 an over plot of two disk spectra in both the EUV and FUV. The first spectrum in Figure 15 in each channel is in black and represents a disk spectrum in the range of $90-110^{\circ} \mathrm{SZA}\left(100^{\circ}\right.$ in plot caption) called a dark disk spectrum versus a second spectrum disk spectrum in range $110^{\circ}-130^{\circ}\left(120^{\circ}\right.$ in plot caption $)$ also called a dark disk spectrum. The two disk spectra are nearly identical in intensity.

[51] The starting point for the regression fitting procedure in the FUV requires fitting the particle scattering vector in the $1500-1880 \mathrm{~A}$ region to get the mixing ratios of $\mathrm{C}_{2} \mathrm{H}_{2}$, haze, $\mathrm{C}_{2} \mathrm{H}_{4}$ and $\mathrm{C}_{4} \mathrm{H}_{2}$. We find the mixing ratios of hydrocarbons relative to $\mathrm{N}_{2}$ to be: $3.9 \times 10^{-6}$ for $\mathrm{C}_{2} \mathrm{H}_{2}, 9.7 \times 10^{-8}$ for $\mathrm{C}_{2} \mathrm{H}_{4}, \sim 0$ for $\mathrm{C}_{4} \mathrm{H}_{2}$ and $9.8 \times 10^{-11}$ for haze, exactly as we 

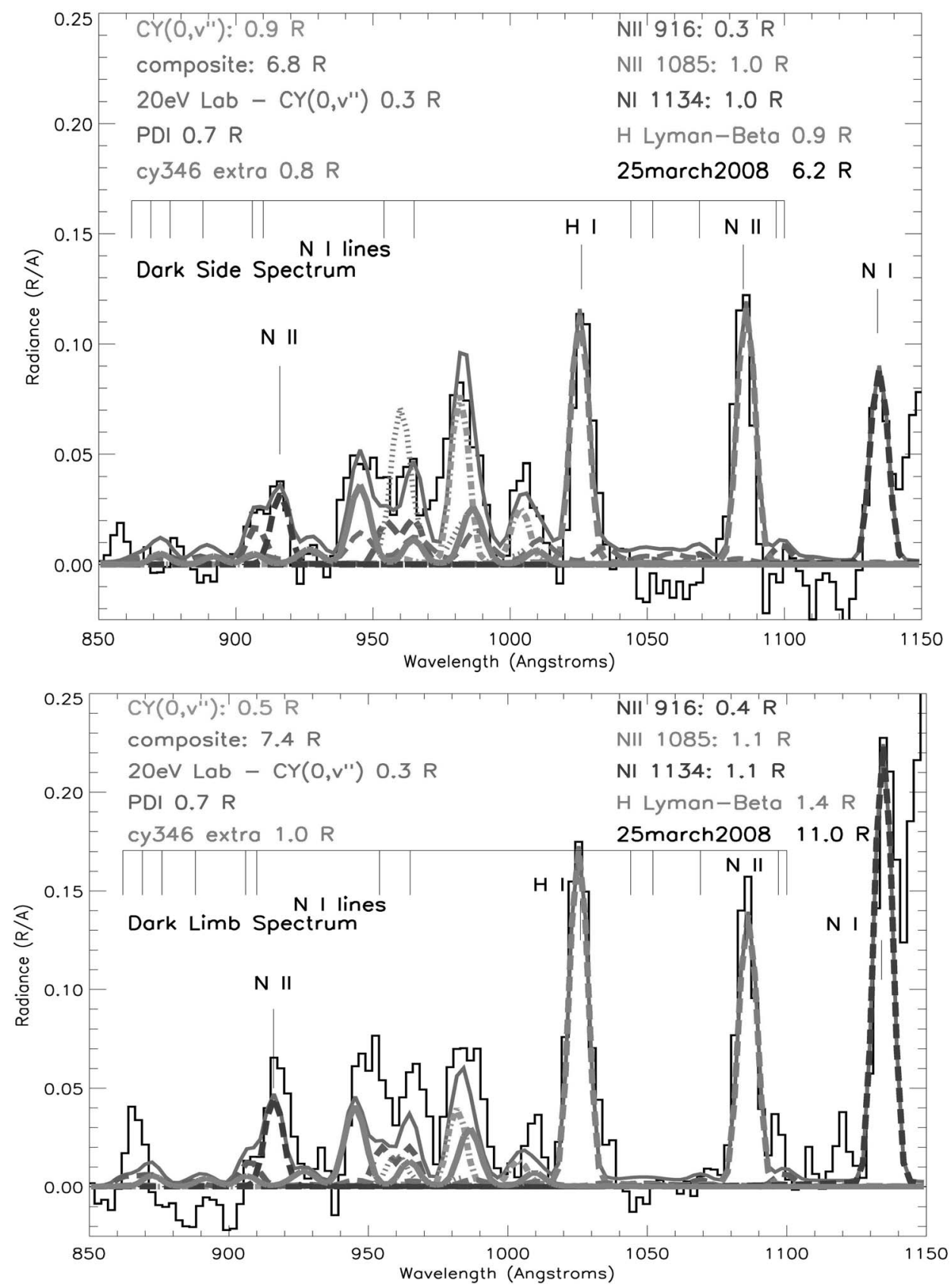

Figure 14. (top) Model fit to UVIS dark side limb EUV spectrum (black) from 25 March 2008. The regression model composite fit (solid green) includes seven independent vectors (totals indicated by color; see text). The optically thin $c_{4}^{\prime}(0) \rightarrow \mathrm{X}(0)$ laboratory band that was removed from vector 1 of $\mathrm{CY}$ bands (laboratory $20 \mathrm{eV}$ spectrum) is shown in dotted-yellow and is replaced by a model described in Papers 1 and 3. The optically thick $c_{4}^{\prime}(0) \rightarrow \mathrm{X}\left(\mathrm{v}^{\prime \prime}=0,1, \ldots\right)$ bands are calculated as described in the text (Papers 1 and 3) and are in blue, and the loss in area of the $c_{4}^{\prime}(0,0)$ band contributes multiplescattering predissociation of $\mathrm{N}_{2}$. The optically thin laboratory spectrum (orange) was used to model the remainder of the $\mathrm{N}_{2}$ electronic band systems which are optically thin; the atomic lines are modeled in green, yellow and blue as described in Paper 3 [see also Samson et al., 1991; Meier et al., 1991; Bishop and Feldman., 2003; Bishop et al., 2007]; and the CY v' =3, 4, 6 bands in purple are modeled as described in Paper 3. (bottom) Dark limb data and regression model as in Figure 14 (top).

did in Paper 2. In Paper 2 we found similar mixing ratios of hydrocarbons relative to $\mathrm{N}_{2}$ to be: $2.4 \times 10^{-6}$ for $\mathrm{C}_{2} \mathrm{H}_{2}$, $5.7 \times 10^{-8}$ for $\mathrm{C}_{2} \mathrm{H}_{4}, 1.2 \times 10^{-8}$ for $\mathrm{C}_{4} \mathrm{H}_{2}$. Our abundance ratios sample all the altitudes above $300 \mathrm{~km}$, i.e., the altitudes where the reflected solar flux is contributing to the FUV Titan spectrum [Wilson and Atreya, 2004]. Moreover, each individual FUV pixel probes all the layers that cross along the line of sight. The retrieved mixing ratio thus cannot 

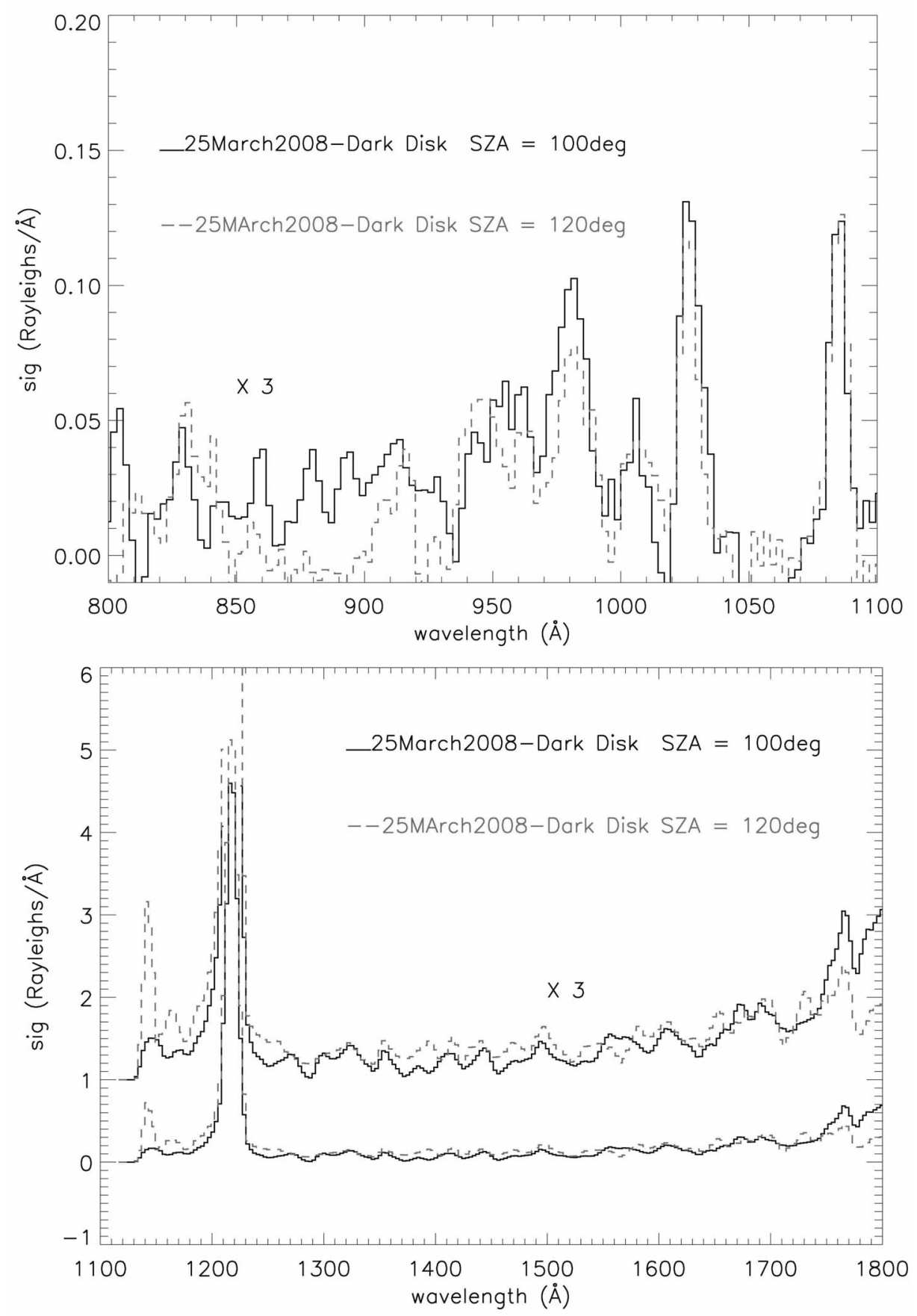

Figure 15. (top) The EUV disk spectrum from 25 March 2008 at $100^{\circ}\left(90-110^{\circ}\right)$ SZA (black) and 120 $\left(110-130^{\circ}\right)$ SZA (dash-orange). (bottom) The FUV disk spectrum from 25 March 2008 at $100^{\circ}$ SZA (black) and $120^{\circ}$ SZA (dash-orange).

be readily compared to other measurements. A significant latitudinal variability has also been noted by Vinatier et al. [2007], which makes the comparison even more difficult.

[52] We then use these mixing ratios with absorption cross sections to derive the reflected sunlight for the whole UVIS band pass. We used a TIMED SEE solar spectrum from 2008 DOY87 [Lean et al., 2011; Kameta et al., 2002]. The sun that day at earth orbit is the same as that which was seen by Titan on DOY 085 . The regression fits, as described earlier, to the FUV spectra for the twilight disk and twilight limb are shown in Figure 16. The spectra contain solar scattering at a low level compared to bright disk and bright limb (not shown).

\section{Summary}

[53] The 1-yr set of observations, studied in this work, occurred mostly in 2009 and allow varied geometry e.g., the spacecraft distances to Titan change from $10^{4}$ to $10^{6} \mathrm{~km}$, the MRH values vary from 0 to $7000 \mathrm{~km}$, the SZA values vary 

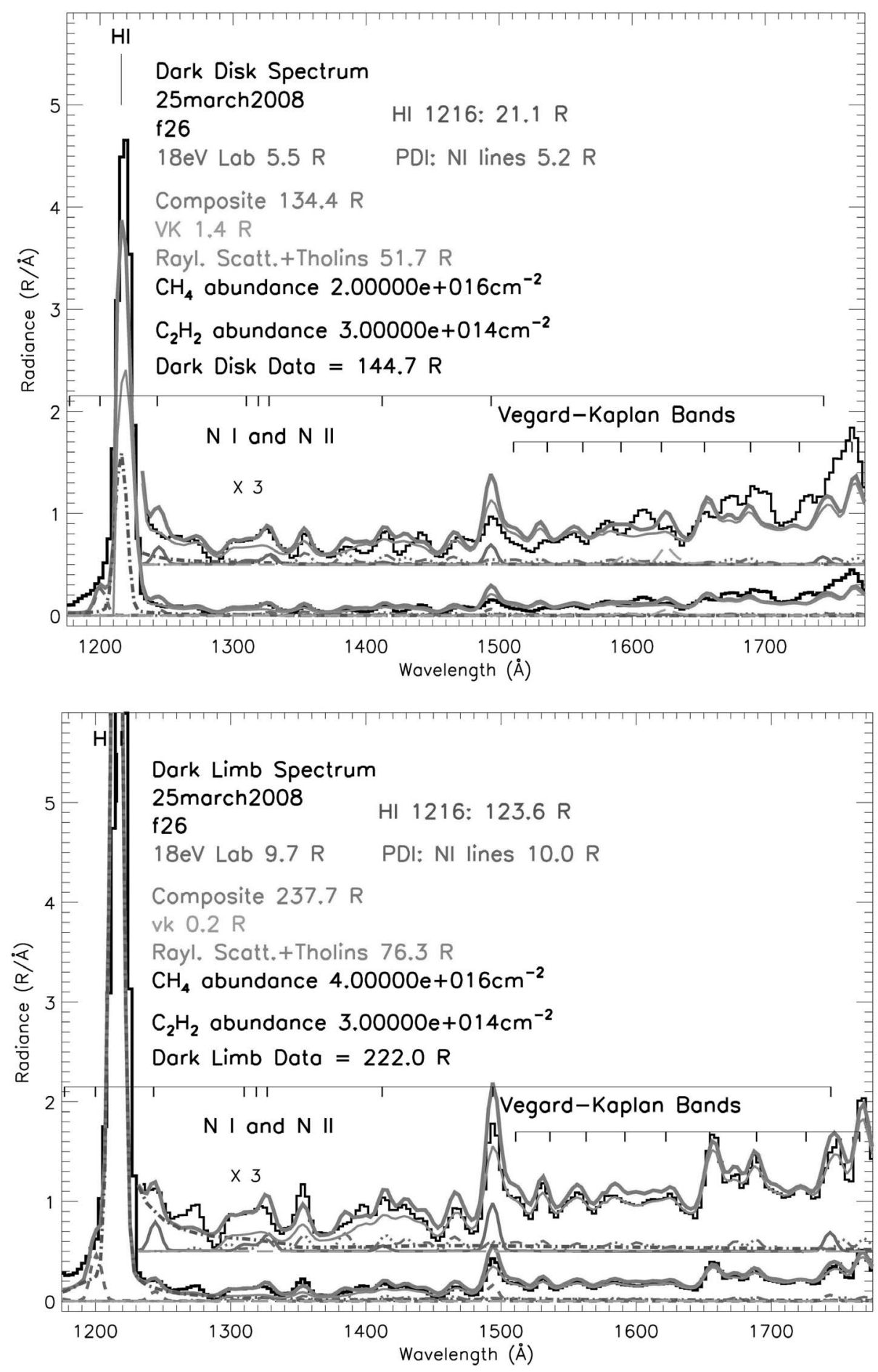

Figure 16. (top) Regression model fit to the FUV twilight glow disk spectrum from 25 Mar 2008 from 1175 to $1775 \AA$ with a particle scattering model described in Paper 2. The model used a TIMED SEE solar spectrum from 2008 DOY87 for the solar reflection component. The regression model (orange) includes four independent spectra: 1) LBH spectrum in red (see text); 2) PDI spectrum from dissociative ionization of $\mathrm{N}_{2}$ in purple; 3) the Rayleigh scattered component described in Figure 15 in blue; and 4) the H Ly $\alpha$ feature at $1216 \AA$ in green. (bottom) Twilight glow data and regression model as in Figure 16 (top). The modeling parameters for $\mathrm{C}_{2} \mathrm{H}_{2}$ and $\mathrm{CH}_{4}$ column densities are shown. The spectral component intensities of the four independent variables and composite spectrum are listed. 
from $50^{\circ}$ to $150^{\circ}$. The altitude of peak UV emission on the limb of Titan during daylight occurred inside the thermosphere at the altitude of the ionosphere (near $1000 \mathrm{~km}$ altitude). This altitude layer has a sunset into total darkness at $\sim 120^{\circ}$ SZA.

[54] The 71 airglow observations analyzed in this paper

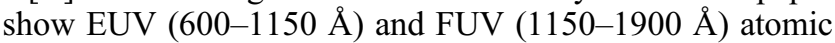
multiplet lines and band emissions arising from photoelectron induced fluorescence and solar photo-fragmentation of molecular nitrogen $\left(\mathrm{N}_{2}\right)$ during the dayglow $\left(\mathrm{SZA}<90^{\circ}\right)$ and twilight glow $\left(90^{\circ}<\mathrm{SZA}<120^{\circ}\right)$. We also have identified similar but weaker nightglow emission $\left(\mathrm{SZA}>120^{\circ}\right)$ for the first time on Titan from magnetosphere plasma impact on the $\mathrm{N}_{2}$ atmosphere. A "stare" dayglow observation on 22 June 2009 at $800-1200 \mathrm{~km}$ with SZA of $53^{\circ} \mathrm{MRH}$ produced the highest $\mathrm{S} / \mathrm{N}$ spectrum with the peak airglow region approximately $200 \mathrm{~km} \mathrm{FWHM} \mathrm{in} \mathrm{width} \mathrm{in} \mathrm{altitude.} \mathrm{There} \mathrm{is} \mathrm{a} \mathrm{twi-}$ light glow that persists with solar depression angle for $30^{\circ}$ past the terminator, until the solar XUV shadow height passes the highest altitude of the topside ionosphere near $1200 \mathrm{~km}$. The twilight FUV and EUV spectra are similar to the dayglow spectra but weaker in intensity by a factor of about two. The nightglow spectra are weaker yet by a factor of 3-10 from the dayglow intensities.

[55] The F3 polar nightglow limb observation (20 April 2009) integrated EUV and FUV intensities are about $1 / 3$ the dayglow intensity of the 13 December 2004 and 22 June 2009 observations and appears to be brighter than the F0 equatorial nightglow spectra in the EUV (see Figure 5). More information about the nightglow spectra is obtained by reviewing the 71 observations studied in 2009. The first four data points of Figures 2 and 5, representing F0 though F3 low-altitude $(<1000 \mathrm{~km}$ mean MRH altitudes) LOS observations (Titan encounter T53), indicate nightglow spectra with prominent band and atomic line emission intensities shown in Figure 5. The data points of band and line intensities indicate that F3 was the brightest on the average in the EUV of the four nightglow observations and that the four observations measured FUV intensities that are similar, at least for the LBH bands and the NI $1493 \AA$. Figure 5 shows that F3 was about twice as bright as F0 in the EUV. Also a more interesting perspective is that Figure 5 shows that the LBH intensity and $1493 \AA$ intensity of F14 (22 June 2009) are about 7-8 times brighter than the corresponding F3 or F0 intensities, while in the EUV F14 the $\left.\mathrm{N}_{2} c_{4}^{\prime 1} \sum_{u}^{+}\right)(0,1)$ band at $980 \AA$, and the N II multiplet $\left(\mathrm{g}^{3} \mathrm{P}_{-}{ }^{3} \mathrm{P}^{\mathrm{o}}\right)$ near $1085 \AA$ are only three times brighter than the corresponding intensities in F3.

[56] Beyond $120^{\circ} \mathrm{SZA}$, when the upper atmosphere of the moon is in total darkness, there are UV emissions from plasma impact of charged particles [Cravens et al., 2008] over an extended altitude from 500 to $1200 \mathrm{~km}$. The weak emissions are variable and occur at about $10-20 \%$ of the level of the dayglow. First in the EUV at the distance of $600,000 \mathrm{~km}$ as in the eclipse, we perceive three strong features in the EUV: the $c_{4}^{\prime}(0,1)$ band at $980 \AA$, the $\mathrm{H} \mathrm{Ly} \beta$ line at $1025 \AA$, the N II $1085 \AA$ multiplet in the EUV and several N I multiplets at $1200 \AA, 1243 \AA$ and $1493 \AA$ with a weak $N_{2}$ LBH band system in the FUV. However, at the closest approach Titan-spacecraft distance of F0 and F3 ( 20,000 km) of T53 we see additional strong features in the EUV and FUV with filled FOV for each pixel. We find the $c_{4}^{\prime}(3,2)$ and $c_{4}^{\prime}(4,3)$ bands at $945 \AA$, the $c_{4}^{\prime}(0,2)$ band at $1003 \AA$, the N I multiplet at $1134 \AA$ and the N II $917 \AA$ multiplet. These features are difficult to see in the weak eclipse spectrum. In the FUV we find the VK and LBH bands. The latter emission set particularly the VK system and the NI $1493 \AA$ multiplet appears to be excited from below $800 \mathrm{~km} \mathrm{limb}$ ray height to a level originating from lower thermosphere, mesosphere, haze layer extending downward to $\sim 500 \mathrm{~km}$. These emissions possibly result from proton- and/or heavier ion-induced emissions as well as secondary-electron-induced emissions. The depth of penetration is consistent with the models of Wilson and Atreya [2004] that show particle impact becomes important below $1000 \mathrm{~km}$, providing an important source in the lower ionosphere region.

[57] The eclipse observations are unique and have been reported in a recent pair of publications [West et al., 2011, 2012]. UV emissions were observed during only one of the three eclipse events, and only weak LBH and Vegard-Kaplan (VK) emissions were seen in the FUV. The F0 and F3 observations show the advantage of the close-encounter and low altitude MRH 500-1000 km for high S/N observations for Titan dark side measurements of the FUV emissions of the $\mathrm{VK}$ and LBH band systems. Near-polar observations may be more intense in the UV as a complementary result to strong polar lower atmosphere electron density profiles as illustrated by Cravens et al. [2009] and Kliore et al. [2008]. The darkest (no particle scattering) FUV limb and disk spectra that include a UV slant path contribution from the lower ionosphere layer occur during the 7 May 2009 eclipse. These UV eclipse spectra, which show low signal intensity, will be useful in putting an upper limit to the role of magnetosphere plasma excitation.

[58] We have employed regression analysis using laboratory spectra of the 25 March 2008 UVIS Titan observation, which included four types of measurements (bright and dark disk and bright and dark (twilight) limb); and we have analyzed the intensities and identified each spectral feature from the limb or disk emission spectrum. The $\mathrm{N}$ I multiplets, $\mathrm{N}_{2} \mathrm{VK}$ and $\mathrm{N}_{2}$ LBH bands are prominent in the twilight glow until $120^{\circ} \mathrm{SZA}$. The details of the spectral content are given in Papers 2 and 3. We plan further work to model magnetosphere plasma energy input to the nighttime airglow emissions.

[59] Acknowledgments. This work was performed at the Jet Propulsion Laboratory (JPL), California Institute of Technology (Caltech), under a contract with the National Aeronautics and Space Administration (NASA). We gratefully acknowledge financial support through NASA's Cassini Data Analysis Program, Geospace and Planetary Atmospheres programs, the National Science Foundation, and the Astrophysics Research and Analysis Program Offices.

[60] Robert Lysak thanks the reviewers for their assistance in evaluating this paper.

\section{References}

Ajello, J. M., and B. Franklin (1985), A study of the extreme ultraviolet spectrum of $\mathrm{O}_{2}$ by electron impact, J. Chem. Phys., 82, 2519, doi:10.1063/1.448301

Ajello, J. M., and D. E. Shemansky (1985), A reexamination of important $\mathrm{N}_{2}$ cross sections by electron impact with application to the dayglow: The Lyman-Birge-Hopfield band system and N I (119.99 nm), J. Geophys. Res., 90, 9845-9861, doi:10.1029/JA090iA10p09845.

Ajello, J. M., C. W. Hord, C. A. Barth, A. I. Stewart, and A. L. Lane (1973), Mariner 9 Ultraviolet Spectrometer Experiment: Afternoon terminator observations of Mars, J. Geophys. Res., 78(20), 4279-4290, doi:10.1029/ JB078i020p04279. 
Ajello, J. A., G. K. James, B. O. Franklin, and D. E. Shemansky (1989), Medium-resolution studies of extreme ultraviolet emission from $\mathrm{N}_{2}$ by electron impact: Vibrational perturbations and cross sections of the $\mathrm{c}^{\prime}{ }_{4}^{1} \sum_{\mathrm{u}}^{+}$and $\mathrm{b}^{\prime 1} \sum_{\mathrm{u}}^{+}$states, Phys. Rev. A, 40, 3524-3556, doi:10.1103/ PhysRevA.40.3524.

Ajello, J. M., G. K. James, and M. Ciocca (1998), High-resolution EUV emission spectroscopy of the $\mathrm{N}_{2} \mathrm{c}^{\prime}{ }^{1} \sum_{\mathrm{u}}^{+} \mathrm{v}^{\prime}=3$ and 4 levels by electron impact, J. Phys. B At. Mol. Opt. Phys., 31, 2437-2448, doi:10.1088/ 0953-4075/31/10/028

Ajello, J. M., et al. (2007), Titan airglow spectra from Cassini Ultraviolet Imaging Spectrograph (UVIS): EUV analyses, Geophys. Res. Lett., 34, L24204, doi:10.1029/2007GL031555.

Ajello, J. M., et al. (2008), Titan airglow spectra from the Cassini Ultraviolet Imaging Spectrograph: FUV disk analysis, Geophys. Res. Lett., 35, L06102, doi:10.1029/2007GL032315.

Ajello, J. M., R. S. Mangina, and R. R. Meier (2010), UV molecular spectroscopy from electron impact for applications to planetary atmospheres and astrophysics, in Charged Particle and Photon Interactions With Matter: Recent Advances, Applications, and Interfaces, edited by Y. Hatano, Y. Katsumura, and A. Mozumder, pp. 761-804, CRC Press, Boca Raton, Fla., doi:10.1201/b10389-29.

Ajello, J. M., R. A. West, C. P. Malone, J. Gustin, L. W. Esposito, W. E. McClintock, G. M. Holsclaw, and M. H. Stevens (2011a), Cassini UVIS observations of Titan ultraviolet airglow spectra with laboratory modeling from electron- and proton-excited N2 emission studies, Abstract SA11A05, presented at 2011 Fall Meeting, AGU, San Francisco, Calif., 5-9 Dec. Ajello, J. M., R. Mangina, D. Strickland, and D. Dziczek (2011b), Laboratory studies of UV emissions from proton-impact on $\mathrm{N}_{2}$ : The LymanBirge-Hopfield band system for aurora analysis, J. Geophys. Res., 116, A00K03, doi:10.1029/2010JA016103.

Bishop, J., and P. D. Feldman (2003), Analysis of the Astro-1/Hopkins Ultraviolet Telescope EUV-FUV dayside nadir spectral radiance measurements, J. Geophys. Res., 108(A6), 1243, doi:10.1029/2001JA000330.

Bishop, J., M. H. Stevens, and P. D. Feldman (2007), Molecular nitrogen Carroll-Yoshino $\mathrm{v}^{\prime}=0$ emission in the thermospheric dayglow as seen by the Far Ultraviolet Spectroscopic Explorer, J. Geophys. Res., 112, A10312, doi:10.1029/2007JA012389.

Broadfoot, A. L., et al. (1997), $\mathrm{N}_{2}$ triplet band systems and atomic oxygen in the dayglow, J. Geophys. Res., 102, 11,567-11,584, doi:10.1029/ 97JA00771.

Cravens, T. E., I. P. Robertson, S. A. Ledvina, D. S. M. Mitchell, and S. M. Krimigis (2008), Energetic ion precipitation at Titan, Geophys. Res. Lett., 35, L03103, doi:10.1029/2007GL032451.

Cravens, T. E., R. V. Yelle, J.-E. Wahlund, D. E. Shemansky, and A. F. Nagy (2009), Composition and structure of the Ionosphere and thermosphere, in Titan From Cassini-Huygens, edited by R. Brown, J.-P Lebreton, and H. Waite, pp. 259-295, Springer, Dordrecht, Netherlands, doi:10.1007/978-1-4020-9215-2 11.

De La Haye, V., et al. (2007), Cassini Ion and Neutral Mass Spectrometer data in Titan's upper atmosphere and exosphere: Observation of a suprathermal corona, J. Geophys. Res., 112, A07309, doi:10.1029/2006JA012222.

Esposito, L. W., et al. (2004), The Cassini Ultraviolet Imaging Spectrograph investigation, Space Sci. Rev., 115, 299-361, doi:10.1007/ s11214-004-1455-8.

Feldman, P. D., D. J. Sahnow, J. W. Kruk, E. M. Murphy, and H. Warren Moos (2001), High-resolution FUV spectroscopy of the terrestrial day airglow with the Far Ultraviolet Spectroscopic Explorer, J. Geophys. Res., 106, 8119-8129, doi:10.1029/2000JA000356.

Fulchignoni, M., et al. (2005), In situ measurements of the physical characteristics of Titan's environment, Nature, 438, 785-791, doi:10.1038/ nature04314.

Gan, L., C. N. Keller, and T. E. Cravens (1992), Electrons in the ionosphere of Titan, J. Geophys. Res., 97, 12,137-12,151, doi:10.1029/92JA00300.

Gladstone, G. R., S. A. Stern, and W. R. Pryor (2012, in press), New Horizons cruise observations of Lyman- $\alpha$ emissions from the interplanetary medium, in Cross-Calibration of Far UV Spectra of Solar System Objects and the Heliosphere, ISSI Sci. Rep. Ser., vol. 13, edited by E. Quémerais, M. Snow, and R.-M. Bonnet, pp. 175-188, Int. Space Sci. Inst., Bern, Switzerland

Kameta, K., et al. (2002), Photoabsorption, photoionization, and neutraldissociation cross sections of simple hydrocarbons in the vacuum ultraviolet range, J. Electron Spectrosc. Relat. Phenom., 123, 225-238, doi:10.1016/S0368-2048(02)00022-1.

Kliore, A. J., et al. (2008), First results from the Cassini radio occultations of the Titan ionosphere, J. Geophys. Res., 113, A09317, doi:10.1029/ 2007JA012965.

Lavvas, P., M. Galand, R. V. Yelle, A. N. Heays, B. R. Lewis, G. R. Lewis, and A. J. Coates (2011), Energy deposition and primary chemical products in Titan's upper atmosphere, Icarus, 213(1), 233-251, doi:10.1016/ j.icarus.2011.03.001.

Lean, J. T., T. N. Woods, F. G. Eparvier, R. R. Meier, D. J. Strickland, J. T. Correira, and J. S. Evans (2011), Solar extreme ultraviolet irradiance: Present, past and future, J. Geophys. Res., 116, A01102, doi:10.1029/ 2010JA015901.

Ledvina, S. A., T. E. Cravens, and K. Keckemety (2005), Ion distribution in Saturn's magnetosphere near Titan, J. Geophys. Res., 110, A06211, doi:10.1029/2004JA010771

Lewis, B. R., et al. (2005a), Lifetime and predissociation yield of ${ }^{14} \mathrm{~N}_{2}$ $\mathrm{b}^{1} \Pi_{\mathrm{u}}(\mathrm{v}=1)$ revisited: Effects of rotation, J. Chem. Phys., 123, 236101, doi:10.1063/1.2137722.

Lewis, B. R., et al. (2005b), Predissociation mechanism for the lowest ${ }^{1} \Pi_{4}$ states of $\mathrm{N}_{2}$, J. Chem. Phys., 122, 144302, doi:10.1063/1.1869986.

Lewis, B. R., et al. (2008a), Optical observation of the C, $3 \mathrm{~s} \sigma_{\mathrm{g}} \mathrm{F}_{3}$, and $3 \mathrm{p} \pi_{\mathrm{u}} \mathrm{G}_{3}{ }^{3} \Pi_{\mathrm{u}}$ states of $\mathrm{N}_{2}, J$. Chem. Phys., 129, 164305, doi:10.1063/ 1.2990655 .

Lewis, B. R., et al. (2008b), A coupled-channel model of the ${ }^{3} \Pi_{u}$ states of $\mathrm{N}_{2}$ : Structure and interactions of the $3 \mathrm{~s} \sigma_{\mathrm{g}} \mathrm{F}_{3}^{3} \Pi_{\mathrm{u}}$ and $3 \mathrm{p} \pi_{\mathrm{u}} \mathrm{G}_{3}{ }^{3} \Pi_{\mathrm{u}}$ Rydberg states, J. Chem. Phys., 129, 164306, doi:10.1063/1.2990656.

Liu, X., D. E. Shemansky, M. Ciocca, I. Kanik, and J. M. Ajello (2005), Analysis of the physical properties of the $\mathrm{N}_{2} c^{\prime 1} \sum_{\mathrm{u}}^{+}(0)-X^{1} \sum_{g}^{+}(0)$ transition, Astrophys. J, 623, 579-584, doi:10.1086/428641.

Liu, X., D. E. Shemanksy, C. Malone, P. Johnson, J. M. Ajello, I. Kanik, A. Heays, B. Lewis, S. Gibson, and G. Stark (2008), Experimental and coupled channels investigation of the radiative properties of the $\mathrm{N}_{2} \mathrm{c}^{\prime}$ ${ }^{1} \sum_{\mathrm{u}^{+}}^{+} \mathrm{X} \sum_{\mathrm{g}}^{+}$band system, J. Geophys. Res., 113, A02304, doi:10.1029/ 2007JA012787.

Mangina, R. S., J. M. Ajello, and G. K. James (2011), High-resolution electron-impact emission spectra and vibrational emission cross sections from 330-1100 nm for $\mathrm{N}_{2}$, Astrophys. J., 196(1), 13, doi:10.1088/0067-0049/ 196/1/13.

Meier, R. R., J. A. R. Samson, Y. Chung, E.-M. Lee and Z.-X. He (1991), Production of $\mathrm{N}^{+} *$ from $\mathrm{N}_{2}+h \nu$ : Effective EUV emission yields from laboratory and dayglow data, Planet. Space Sci., 39, 1197-1207, doi:10.1016/0032-0633(91)90171-6.

Melin, H., D. E. Shemansky, and X. Liu (2009), The distribution of atomic hydrogen and oxygen in the magnetosphere of Saturn, Planet. Space Sci. 438(14-15), 1743-1753, doi:10.1016/j.pss.2009.04.014.

Morrison, M. D., C. W. Bowers, and P. D. Feldman (1990), The EUV dayglow at high spectral resolution, J. Geophys. Res., 95, 4113-4127, doi:10.1029/ JA095iA04p04113.

Mumma, M. J., and E. C. Zipf (1971), Dissociative excitation of vacuumultraviolet emission features by electron impact on molecular gases, 2, $\mathrm{N}_{2}$, J. Chem. Phys., 55, 5582-5588, doi:10.1063/1.1675725.

Roncin, J. Y., J. L. Subtil, and F. Launay (1998), The high-resolution vacuum ultraviolet emission spectrum of molecular nitrogen from 82.6 to $124.2 \mathrm{~nm}$ : Level energies of 10 excited singlet electronic states, J. Mol. Spectrosc., 188, 128-137, doi:10.1006/jmsp.1997.7497.

Rymer, A. M., H. T. Smith, A. Weellbrock, A. J. Coaters, and D. T. Young (2009), Discrete classification and electron energy spectra of Titan's varied magnetospheric environment, Geophys. Res. Lett., 36, L15109, doi:10.1029/2009GL039427.

Samson, J. A. R., Y. Chung, and E.-M. Lee (1991), Excited ionic and neutral fragments produced by dissociation of the $\mathrm{N}_{2}^{+} * \mathrm{H}$ band, J. Chem. Phys., 95, 717-719, doi:10.1063/1.461424

Shah, M. B., C. J. Latimer, E. C. Latimer, E. C. Montenegro, O. J. Tucker, R. E. Johnson, and H. T. Smith (2009), The implantation and interactions of $\mathrm{O}^{+}$in Titan's atmosphere: Laboratory measurements of collisioninduced dissociation of $\mathrm{N}_{2}$ and modeling of positive ion formation, Astrophys. J., 703, 1947-1954, doi:10.1088/0004-637X/703/2/1947.

Shemansky, D. E. (1969), $\mathrm{N}_{2}$ Vegard-Kaplan system in absorption, J. Chem. Phys., 51, 689-700, doi:10.1063/1.1672058.

Shemansky, D. E., I. Kanik and J. M. Ajello (1995), Fine-structure branching in $\mathrm{N}_{2} c^{\prime}{ }_{4}^{1} \sum_{\mathrm{u}}^{+}(0)$, Astrophys. J., 452, 480-485, doi:10.1086/176320.

Shemansky, D., I. Stewart, R. West, L. Esposito, J. Hallett, and X. Liu (2005), The Cassini UVIS stellar probe of the Titan atmosphere, Science, 308, 978, doi:10.1126/science. 1111790 .

Sittler, E. C., R. E. Hartle, C. Bertucci, A. Coates, T. E. Cravens, I. Dandouras, and D. Shemansky (2010a), Energy deposition processes in Titan's upper atmosphere and its induced magnetosphere, in Titan From Cassini-Huygens, edited by R. Brown, J.-P. Lebreton, and H. Waite, pp. 393-454, Springer, Dordrecht, Netherlands

Sittler, E. C., et al. (2010b), Saturn's magnetospheric interactions with Titan as defined by Cassini encounters T9 and T18: New results, Planet. Space Sci., 58, 327-350, doi:10.1016/j.pss.2009.09.017.

Smith, H. T., D. G. Mitchell, R. E. Johnson, and C. P. Paranicas (2009), Investigation of energetic proton penetration in Titan's atmosphere using 
the Cassini INCA instrument, Planet. Space Sci., 57, 1538-1546, doi:10.1016/j.pss.2009.03.013.

Stevens, M. H. (2001), The EUV airglow of Titan: Production and loss of $\mathrm{N}_{2} \quad c^{\prime}{ }_{4}(0)-X, \quad J . \quad$ Geophys. Res., 106, 3685-3689, doi:10.1029/ 1999JA000329.

Stevens, M. H., et al. (2011), The production of Titan's ultraviolet nitrogen airglow, J. Geophys. Res., 116, A05304, doi:10.1029/2010JA016284.

Strobel, D., and J. Cui (2012 in press), Titan's upper atmosphere/exosphere, escape processes, and rates, in Titan: Interior, Surface, Atmosphere, and Space Environment, Cambridge Planet. Sci. Ser., vol. 14, edited by I. Müller-Wodarg et al., pp. 279-295, Cambridge Univ. Press, Cambridge, U. K.

Tomasko, M. G., and R. A. West (2010), Aerosols in Titan's atmosphere, in Titan From Cassini-Huygens, edited by R. Brown, J.-P. Lebreton, and H. Waite, pp. 297-321, Springer, Dordrecht, Netherlands, doi:10.1007/ 978-1-4020-9215-2 12

Ubachs, W., et al. (2001), Lifetime measurements on the $\mathrm{c}^{\prime}{ }_{4}^{1} \sum_{u}^{+}, v=0,1$ and 2 states of molecular nitrogen, Chem. Phys., 270, 215-225, doi:10.1016/S0301-0104(01)00378-0.
Vinatier, S., et al. (2007), Vertical abundance profiles of hydrocarbons in Titan's atmosphere at $15^{\circ} \mathrm{S}$ and $80^{\circ} \mathrm{N}$ retrieved from Cassini/CIRS spectra, Icarus, 188(1), 120-138, doi:10.1016/j.icarus.2006.10.031.

West, R. A., J. M. Ajello, M. H. Stevens, J. S. Evans, and R. S. Mangina (2011), Airglow emission from Titan during eclipse, paper presented at EPSC-DPS Joint Meeting 2011, Nantes, France, 2-7 Oct.

West, R. A., J. M. Ajello, M. H. Stevens, D. F. Strobel, G. R. Gladstone, J. S. Evans, and E. T. Bradley (2012), Titan airglow during eclipse, Geophys. Res. Lett., 39, L18204, doi:10.1029/2012GL053230.

Westlake, J. H., J. M. Bell, J. H. Waite, R. E. Johnson, J. G. Luhmann, K. E. Mandt, B. A. Magee, and A. M. Rymer (2011), Titan's thermospheric response to various plasma environments, J. Geophys. Res., 116, A03318, doi:10.1029/2010JA016251.

Wilson, E. H. W., and S. K. Atreya (2004), Current state of modeling the photochemistry of Titan's mutually dependent atmosphere and ionosphere, J. Geophys. Res., 109, E06002, doi:10.1029/2003JE002181.

Young, J. A., et al. (2010), Lyman-Birge-Hopfield emissions from electronimpact excited $\mathrm{N}_{2}$, J. Phys. B At. Mol. Opt. Phys., 43, 135201, doi:10.1088/0953-4075/43/13/135201. 